\title{
KAC-WARD OPERATORS, KASTELEYN OPERATORS, AND S-HOLOMORPHICITY ON ARBITRARY SURFACE GRAPHS
}

\author{
DAVID CIMASONI
}

\begin{abstract}
The conformal invariance and universality results of Chelkak-Smirnov on the two-dimensional Ising model hold for isoradial planar graphs with critical weights. Motivated by the problem of extending these results to a wider class of graphs, we define a generalized notion of s-holomorphicity for functions on arbitrary weighted surface graphs. We then give three criteria for s-holomorphicity involving the Kac-Ward, Kasteleyn, and discrete Dirac operators, respectively. Also, we show that some crucial results known to hold in the planar isoradial case extend to this general setting: in particular, spin-Ising fermionic observables are s-holomorphic, and it is possible to define a discrete version of the integral of the square of an s-holomorphic function. Along the way, we obtain a duality result for Kac-Ward determinants on arbitrary weighted surface graphs.
\end{abstract}

\section{INTRODUCTION}

1.1. Motivation. The Ising model is certainly one of the most famous models in statistical physics. It was introduced by Lenz in 1920 [32] as an attempt to understand Curie's temperature for ferromagnets. It can be defined as follows. Let $\Gamma$ be a finite graph with vertex set $V(\Gamma)$ and edge set $E(\Gamma)$. A spin configuration on $\Gamma$ is an element $\sigma$ of $\{-1,+1\}^{V(\Gamma)}$. Given a positive edge weight system $J=\left(J_{e}\right)_{e \in E(\Gamma)}$ on $\Gamma$, the energy of such a spin configuration $\sigma$ is defined by

$$
\mathscr{H}(\sigma)=-\sum_{e=\{u, v\} \in E(\Gamma)} J_{e} \sigma_{u} \sigma_{v}
$$

Fixing an inverse temperature $\beta \geq 0$ determines a probability measure on the set $\Omega(\Gamma)$ of spin configurations by

$$
\mu_{\Gamma, \beta}(\sigma)=\frac{e^{-\beta \mathscr{H}(\sigma)}}{Z_{\beta}^{J}(\Gamma)},
$$

where the normalization constant

$$
Z_{\beta}^{J}(\Gamma)=\sum_{\sigma \in \Omega(\Gamma)} e^{-\beta \mathscr{H}(\sigma)}
$$

is called the partition function of the Ising model on $\Gamma$ with coupling constants $J$. The name of the model comes from a student of Lenz, Ernst Ising, who proved in his PhD Thesis the absence of phase transition in dimension one, i.e. in the case of $\Gamma=\mathbb{Z}$ and $J_{e}$ independent of $e$. He also conjectured the same behavior in higher dimensions, a conjecture later disproved

2000 Mathematics Subject Classification. 82B20, 57M15.

Key words and phrases. Kac-Ward operator, Kasteleyn operator, s-holomorphic functions, Ising model.

This research was partially supported by the Swiss NSF. 
by Peierls [38]. Using their celebrated duality argument, Kramers and Wannier 31] then gave a heuristic derivation of the critical temperature in the case of the square lattice $\Gamma=\mathbb{Z}^{2}$.

The rigorous proof of this later result by Onsager in 1944 together with his exact computation of the partition function [37] led to an explosion of activity in the field. In particular, Kac and Ward 23] tried to find a more combinatorial approach to the results of Onsager. They defined a matrix $K W(\Gamma)$ with rows and columns indexed by the set $\mathbb{E}$ of oriented edges of the square lattice $\Gamma$, whose determinant coincides with the square of $Z^{I s i n g}(\Gamma)$, the high temperature expansion of the Ising partition function on $\Gamma$. However, several arguments in [23] are of heuristic nature, and some key topological statement turned out not to hold [40. Since the technicalities raised by a rigorous proof of this equality seemed formidable, the focus shifted to finding combinatorial methods not involving directly this Kac-Ward matrix. This was achieved independently by Hurst-Green [21, Kasteleyn [25] and Fisher [18]: they related $Z^{\text {Ising }}(\Gamma)$ with the dimer partition function $Z^{\text {dimer }}\left(F_{\Gamma}\right)$ on an associated Fisher graph $F_{\Gamma}$, and found a skew-symmetric adjacency matrix $K\left(F_{\Gamma}\right)$ - the associated Kasteleyn matrix whose Pfaffian gives $Z^{\text {dimer }}\left(F_{\Gamma}\right)$. This method was later extended by Kasteleyn [26] to any planar graph, and by various authors to the general case of surface graphs [41, 20, 11. Note that the first direct combinatorial proof of the Kac-Ward formula for any planar graph was only obtained in 1999 by Dolbilin et al. [14, and recently extended by the author to surface graphs [7].

There are several issues with this Fisher correspondence $\Gamma \mapsto F_{\Gamma}$, the main one being that it does not preserve crucial geometric and combinatorial properties of the graph. For example, if $\Gamma$ is an isoradial graph, i.e. if each face is inscribed into a circle of fixed radius, then $F_{\Gamma}$ will not be isoradial in general. Also, even if $\Gamma$ is bipartite, i.e. if its vertices split up into two sets $B(\Gamma) \cup W(\Gamma)$ such that the edges never link vertices in the same set, $F_{\Gamma}$ will not be bipartite. This is a problem, since many remarkable statements about the dimer model are known to hold only for bipartite [29] or isoradial graphs [27]... and yet, they did seem to transfer to the Ising model [2, 3]. The explanation to this mystery came in the recent paper of Dubédat [15]: he (re)discovered another mapping $\Gamma \mapsto C_{\Gamma}$ [44, where this time, $C_{\Gamma}$ is always bipartite, and always isoradial if $\Gamma$ is. This new mapping therefore permits the transfer of the whole power of the dimer technology to the Ising model.

As apparent from its definition, the Ising model can be studied on an arbitrary abstract weighted graph $(\Gamma, J)$. However, as explained above, the effective computation of its partition function requires the choice of an embedding of $\Gamma$ in a surface. Furthermore, at criticality, the coupling constants $J$ are expected to correspond to a natural embedding of $\Gamma$ such that the model exhibits properties of conformal invariance and universality at the scaling limit. This can be stated in a precise way in (at least) two different settings: the isoradial and biperiodic cases. If the graph is isoradially embedded in the plane, then there are natural coupling constants such that the model is critical at inverse temperature $\beta=1$. (The first rigorous proof of this fact was obtained recently by Lis [35] using Kac-Ward matrices.) Note that graphs can be isoradially embedded in flat surfaces of arbitrary genus (with cone-type singularities), and that such a flat metric defines a conformal structure on the surface (see [42]). Therefore, it does make sense to talk about critical embeddings of graphs in surfaces of arbitrary topology (a situation first considered by Mercat [36]), and to conjecture conformal invariance and universality of the model at the scaling limit. For example, it is believed that at the critical 
temperature and in the scaling limit, the Ising partition function satisfies

$$
\log Z_{\beta_{c}}^{J}(\Gamma) \simeq f|V(\Gamma)|+h \log |V(\Gamma)|+\mathrm{fsc}
$$

where $f$ is the free energy, $h$ depends on the topology and on the singularities of the metric on $\Sigma$, and fsc is a universal term which only depends on the conformal structure on $\Sigma$. (We refer to [12] for numerical evidence in the case of triangular lattices embedded in a genus 2 surface.) If the graph is planar and biperiodic, i.e. invariant under the action of a lattice $L \simeq \mathbb{Z}^{2}$, then the critical temperature can be determined [33, 10]. (Note that the second reference uses KacWard matrices once again, as well as the correspondence $\Gamma \mapsto C_{\Gamma}$ mentioned above.) Also, the coupling constants determine a natural conformal parameter for the corresponding torus $\mathbb{C} / L$, and once again, it is natural to conjecture conformal invariance and universality at the scaling limit. (See Corollary 3.5 below where we use recent results on the dimer model [30] to check that $\log Z_{\beta}^{J}(\Gamma)$ behaves in the expected way in this setting.)

In the planar isoradial case, a milestone was reached by Smirnov and coauthors in a recent series of papers (see the review [16] and references therein). In particular, Chelkak and Smirnov [6] introduced fermionic observables for the Ising model on any planar critical isoradial graph, and showed that on bounded domains with appropriate boundary conditions, they converge to universal and conformally invariant limits. But why do these authors restrict themselves to planar isoradial graphs, while the universality and conformal invariance are expected to hold at criticality on more general graphs? The problem is that one of the crucial ingredients is a discrete theory of holomorphic functions, and isoradial graphs form the widest class of graphs on which such a theory works sufficiently well [5]. For example, a reasonable discrete version of the $\bar{\partial}$-operator, whose kernel consists of so-called discrete holomorphic functions, does not seem to exist on non-isoradial graphs. Moreover, this notion of discrete holomorphicity does not suffice for the Ising model: Chelkak and Smirnov therefore introduced a stronger notion, known as spin, strong, or simply s-holomorphicity. Among the numerous technical results obtained in [6], we quote the following:

(i) the fact that the fermionic observables are s-holomorphic;

(ii) the possibility to define a discrete version of $h(z)=\operatorname{Im} \int f(z)^{2} d z$ for s-holomorphic functions;

(iii) the subharmonicity and superharmonicity of $h$ on the original graph and on its dual.

In the present paper, we show that, unlike the original notion of discrete holomorphicity, the one of s-holomorphicity does extend to the most general case, that is, to arbitrary weighted graphs embedded in orientable surfaces. Obviously, the whole theory does not extend, but a surprisingly big amount does. Let us also point out that our results do not simply consist in known facts extended from the isoradial to the general case: several statements where previously unknown even in the isoradial case. Therefore, it is our hope that this article will be of interest even to the reader that merely wishes to understand why, in the isoradial case, s-holomorphic functions and fermionic observables are natural objects. Furthermore, our results are particularly appealing not only in the planar isoradial case, but also in the critical biperiodic case and for graphs isoradially embedded in arbitrary surfaces. Therefore, we hope to be able to use them to eventually prove conformal invariance and universality results in these more general settings. 
1.2. Statement of the results. Our results hold for an arbitrary graph $\Gamma$ with edge weights $x \in[0,1]^{E}$ embedded in an orientable surface $\Sigma$. At this level of generality, a geometric tool known as a spin structure is needed, which basically consists in a vector field $\lambda$ on $\Sigma$ with zeroes of even index in $\Sigma \backslash \Gamma$. This allows us to associate to each oriented edge $e \in \mathbb{E}$ an "argument" $a_{e}$ measured with respect to $\lambda$. The cases to keep in mind are the planar and toric ones, where $\lambda$ can be chosen constant; the argument $a_{e}$ then simply gives the direction of $e$, in the sense that the oriented edge $e$ points in the direction of $\exp \left(i a_{e}\right)$ with respect to $\lambda$.

Let us parametrize the edge weight $x_{e} \in[0,1]$ by $x_{e}=\tan \left(\theta_{e} / 2\right)$. Then, we say that a function $F$ defined on the set $\diamond=\left\{z_{e}\right\}_{e \in E}$ of middle points of the edges of $\Gamma$ is s-holomorphic around the vertex $v$ of $\Gamma$ if, for any oriented edge $e \in \mathbb{E}$ originating at $v$,

$\operatorname{Pr}\left(F\left(z_{e}\right) ;\left[i \exp \left(i\left(a_{e}+\theta_{e}\right)\right)\right]^{-\frac{1}{2}}\right)=\operatorname{Pr}\left(F\left(z_{e^{\prime}}\right) ;\left[i \exp \left(i\left(a_{e^{\prime}}-\theta_{e^{\prime}}\right)\right)\right]^{-\frac{1}{2}}\right) \exp \left(\frac{i}{2}\left(\beta_{e}-\theta_{e}-\theta_{e^{\prime}}\right)\right)$, where $\operatorname{Pr}(-; u)$ denotes the orthogonal projection onto $u \cdot \mathbb{R}, e^{\prime} \in \mathbb{E}$ is the edge following $e$ in counterclockwise order around $v$, and $\beta_{e}$ is the oriented angle from $e$ to $e^{\prime}$. (See Definition 6 for details.) Note that in the critical isoradial case, $\beta_{e}-\theta_{e}-\theta_{e^{\prime}}$ vanishes and the equality above defines the usual notion of s-holomorphicity.

Here is one of our main results. (See Theorem 4.2 and Corollary 4.3 for the full statement.)

Theorem. There are explicit $\mathbb{R}$-linear injective maps $S: \mathbb{C}^{\diamond} \rightarrow \mathbb{C}^{\mathbb{E}}$ and $T: \mathbb{C}^{\diamond} \rightarrow \mathbb{C}^{B\left(C_{\Gamma}\right)}$ such that, given $F \in \mathbb{C}^{\diamond}$, the following are equivalent:

(i) $\exp \left(i \frac{\pi}{4}\right) F$ is s-holomorphic.

(ii) $S(F)$ lies in the kernel of the Kac-Ward operator on $\Gamma$.

(iii) $T(F)$ lies in the kernel of the Kasteleyn operator on $C_{\Gamma}$.

Furthermore, if $\Gamma$ is isoradially embedded with critical weights, then there is an explicit $\mathbb{R}$ linear injective map $T^{\prime}: \mathbb{C}^{\diamond} \rightarrow \mathbb{C}^{B\left(C_{\Gamma}\right)}$ such that these conditions are equivalent to:

(iv) $T^{\prime}(F)$ lies in the kernel of the discrete $\bar{\partial}$-operator on $C_{\Gamma}$.

Note that in the planar isoradial case, the equivalence of $(i)$ and $(i i)$ is due to Lis [34. Also, the equivalence of $(i)$ and $(i i i)$ can be understood as a generalization of the "propagation equation" of [6, Section 3.2] (see also [36, Section 4.3] and [15, Section 4.2]).

Our proof of the equivalence of $(i i)$, (iii) and (iv) relies on explicit relations between the corresponding operators (Section (3). We believe that some of these results are of independent interest. In particular, Theorem 3.1, which relates the Kac-Ward operator on $\Gamma$ with the Kasteleyn operator on $C_{\Gamma}$, also immediately implies the following generalized KramersWannier duality. (The original Kramers-Wannier duality corresponds to the planar case.)

Corollary. For any weighted graph $(\Gamma, x) \subset \Sigma$ and any character $\varphi: \pi_{1}(\Sigma) \rightarrow \mathbb{C}^{*}$,

$$
2^{|V(\Gamma)|} \prod_{e \in E(\Gamma)}\left(1+x_{e}\right)^{-1} \operatorname{det}\left(K W^{\varphi}(\Gamma, x)\right)=2^{\left|V\left(\Gamma^{*}\right)\right|} \prod_{e \in E(\Gamma)}\left(1+x_{e}^{*}\right)^{-1} \operatorname{det}\left(K W^{\varphi}\left(\Gamma^{*}, x^{*}\right)\right),
$$

where the weights $x$ and $x^{*}$ are related by $x+x^{*}+x x^{*}=1$.

In the genus one case, this same Theorem 3.1 allows us to transfer the recent results of Kenyon-Sun-Wilson [30] from the dimer to the Ising model. (See Corollary [3.5 below for the full statement.) 
Corollary. Let $(\Gamma, J)$ be a weighted finite toric graph, interpreted as the quotient of a biperiodic planar graph $\mathscr{G}$ by a lattice $L \simeq \mathbb{Z}^{2}$, and set $\Gamma_{n}=\mathscr{G} / n L$. At the critical inverse temperature $\beta=\beta_{c}$, the Ising partition function on $\Gamma_{n}$ satisfies

$$
\log Z_{\beta_{c}}^{J}\left(\Gamma_{n}\right)=n^{2} f\left(\beta_{c}\right)+\mathrm{fsc}(\tau)+o(1),
$$

where $f$ is the free energy per fundamental domain, $\tau \in \mathbb{H}$ can be explicitely computed from $\operatorname{det}\left(K W^{\varphi}\left(\Gamma_{1}, x\right)\right)$, and $\operatorname{fsc}(\tau)$ is a universal function of the modular parameter $\tau$ which is invariant under modular transformations.

Coming back to s-holomorphicity, the theorem stated above should convince the reader that this notion is "natural", as it is linked to the operators appearing in the theory. But is there any non-trivial example of s-holomorphic functions? More precisely, does there exist some generalized fermionic observables that are s-holomorphic? This is the case, and these observables are basically given by the cofactors of the Kac-Ward matrix (a fact obtained independently by Lis [34] in the planar case). For simplicity, we shall only state a corollary and not be very precise with the definitions, referring to Theorem 4.5 for the full statement of the main result, and to subsection 4.3 for more details.

Corollary. Given any fixed oriented edge $e_{0} \in \mathbb{E}$, consider the function $F_{e_{0}} \in \mathbb{C}^{\diamond}$ defined by

$$
F_{e_{0}}(z)=\frac{\exp \left(i \frac{\pi}{4}\right)}{\cos \left(\theta_{e} / 2\right)} \sum_{\xi \in \mathscr{E}\left(e_{0}, z\right)}(-1)^{q_{\lambda}\left(\gamma_{\xi}^{0}\right)} \exp \left(-\frac{i}{2} \operatorname{rot}_{\lambda}\left(\gamma_{\xi}\right)\right) \prod_{e^{\prime} \in \xi} x_{e^{\prime}},
$$

where $z=z_{e} \neq z_{e_{0}}, \mathscr{E}\left(e_{0}, z\right)$ denotes the set of subgraphs $\xi$ consisting in a collection $\gamma_{\xi}^{0}$ of loops together with one path $\gamma_{\xi}$ from $e_{0}$ to $z, \operatorname{rot}_{\lambda}\left(\gamma_{\xi}\right)$ is the total rotation angle of the path $\gamma_{\xi}$ (measured with respect to the fixed vector field $\lambda$ ), and $q_{\lambda}$ is the quadratic form associated with $\lambda$. Then, $F_{e_{0}}$ is s-holomorphic around every vertex of $\Gamma$ not adjacent to $e_{0}$.

This result also has a more direct link to the Ising model. Indeed, consider a biperiodic planar graph $\mathscr{G}$ with edge weights $J=\left(J_{e}\right)_{e} \in(0, \infty)^{E(\mathscr{G})}$. From Theorem 4.5 and [10], we immediately get:

Corollary. There exists a non-trivial biperiodic s-holomorphic function on the weighted graph $(\mathscr{G}, x)$, where $x_{e}=\tanh \left(\beta J_{e}\right)$, if and only if $\beta$ is the critical inverse temperature for the Ising model on $(\mathscr{G}, J)$.

So, many natural functions are s-holomorphic (except possibly at a couple of vertices), and the existence of functions that are s-holomorphic everywhere is related, at least in the case of biperiodic graphs, to criticality.

Finally, we show that the possibility to define a discrete version of $h(z)=\operatorname{Im} \int f(z)^{2} d z$ for s-holomorphic functions does extend to our setting (Proposition 4.9). However, in general, this $h$ is not subharmonic on $\Gamma$ as it is in the critical isoradial case. So this is the point where the extension of the proof of Chelkak-Smirnov breaks down, and where further original ideas are required. We do believe however that the theory of generalized s-holomorphic functions initiated in the present article is a first step in this direction.

1.3. Organisation of the article. In Section 2, we present the main objects involved in the article and settle many notations: weighted surface graphs, isoradial graphs, discrete 
Laplacians, Kac-Ward operators, Kasteleyn operators, and Dirac operators are introduced in this order in separate paragraphs.

In Section 3, we exhibit natural relations between these operators. The most technical such result is Theorem 3.1, which relates Kac-Ward and Kasteleyn operators, implies duality statements (Corollaries 3.3 and 3.4), and allows us to transfer the full dimer technology to the Ising model (Corollary 3.5). The remaining subsections, which only make sense in the isoradial case, relate the Kasteleyn and discrete Dirac operators (Proposition 3.6), the discrete Dirac and Laplace operators (Proposition 3.8), and the discrete Dirac operators on $C_{\Gamma}$ and on the double of $\Gamma$ (Proposition 3.9).

Finally, Section 4 contains our main results. In subsection 4.1, we analyse the kernel of the Kac-Ward operator (Proposition 4.1). In subsection 4.2, we give the definition of s-holomorphicity, and building on the results of Section 3, we prove our three criteria for s-holomorphicity (Theorem 4.2). In subsection 4.3, we analyse the cofactors of the KacWard matrix (Theorem 4.5), thus obtaining the s-holomorphicity of generalized fermionic observables (Corollary 4.7). Finally, in subsection 4.4, we show that a discrete version of the integral of the square of an s-holomorphic function can be defined (Proposition 4.9).

\section{THE GRAPHS AND OPERATORS INVOLVED}

The aim of this section is to present the main objects involved in this article, and for some of them, to recall their role in statistical mechanics. This section is therefore purely expository, and does not contain any new result. It is organised as follows. We first introduce the general setup of weighted surface graphs, presenting in particular the construction of the bipartite graph $C_{\Gamma}$ associated to any surface graph $\Gamma$, which will play a crucial role in this article (subsection 2.11). Then, we recall the setup of isoradial graphs embedded in flat surfaces, a particularly interesting and well-studied class of surface graphs. Finally, in subsections 2.3 to 2.6. we define the operators that will be studied in the rest of the article: the Laplace, KacWard, and Kasteleyn operators - defined on any weighted surface graph - and the discrete Dirac operator, defined only on isoradial graphs.

2.1. Weighted surface graphs. Let us start by setting up our notations for graphs. The set of vertices (resp. edges) of a graph $\Gamma$ will be denoted by $V(\Gamma)$ (resp. $E(\Gamma)$ ). We shall write $\mathbb{E}(\Gamma)$ for the set of oriented edges of $\Gamma$. (Each element of $E(\Gamma)$ thus corresponds to two elements of $\mathbb{E}(\Gamma)$.) Following [39], we shall denote by $o(e)$ the origin of an oriented edge $e \in \mathbb{E}(\Gamma)$, by $t(e)$ its terminus, and by $\bar{e}$ the same edge with the opposite orientation. By abuse of notation, we shall also write $e \in E(\Gamma)$ for the unoriented edge of $\Gamma$ corresponding to the oriented edges $e, \bar{e} \in \mathbb{E}(\Gamma)$. Finally, we shall write $\mathbb{E}(\Gamma)_{v}$ for the set of oriented edges of $\Gamma$ with origin a fixed vertex $v$.

By a weighted surface graph, we mean a finite graph $\Gamma$ endowed with edge weights $x=$ $\left(x_{e}\right)_{e \in E(\Gamma)} \in[0,1]^{E(\Gamma)}$, embedded in a compact connected orientable surface $\Sigma$ so that the complement of $\Gamma$ in $\Sigma$ is the disjoint union of topological discs, which we call faces.

The dual of a weighted surface graph $(\Gamma, x) \subset \Sigma$ is the weighted surface graph $\left(\Gamma^{*}, x^{*}\right) \subset \Sigma$ obtained as follows: each face of $\Gamma \subset \Sigma$ defines a vertex of $\Gamma^{*}$, and each edge of $\Gamma$ bounding two faces of $\Gamma \subset \Sigma$ defines an edge between the two corresponding vertices of $\Gamma^{*}$. More precisely, an oriented edge $e \in \mathbb{E}(\Gamma)$ defines $e^{*} \in \mathbb{E}\left(\Gamma^{*}\right)$, the dual oriented edge obtained by turning 

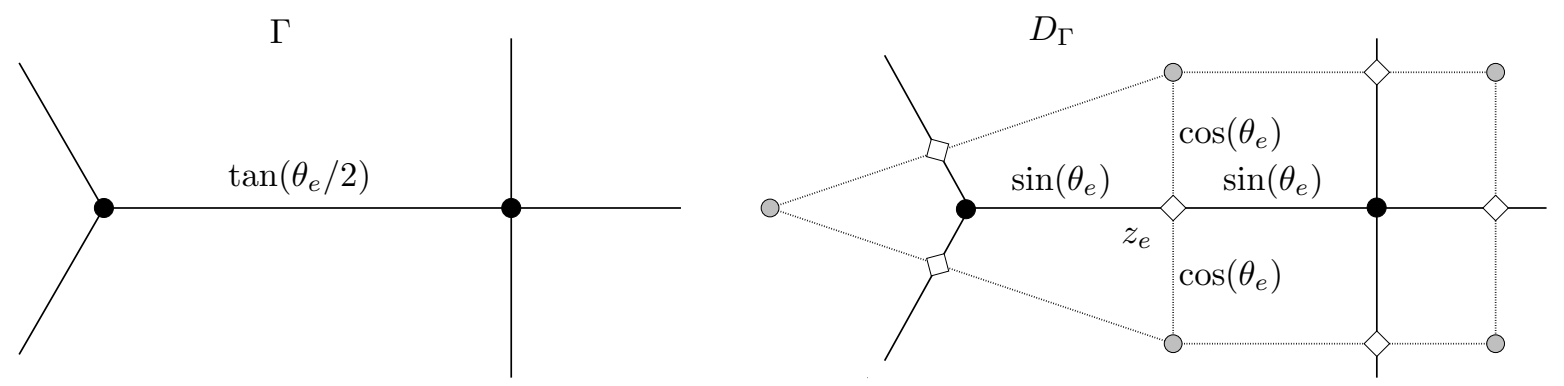

FiguRE 1. The weighted bipartite graph $D_{\Gamma}$ associated to the weighted graph $\Gamma$.
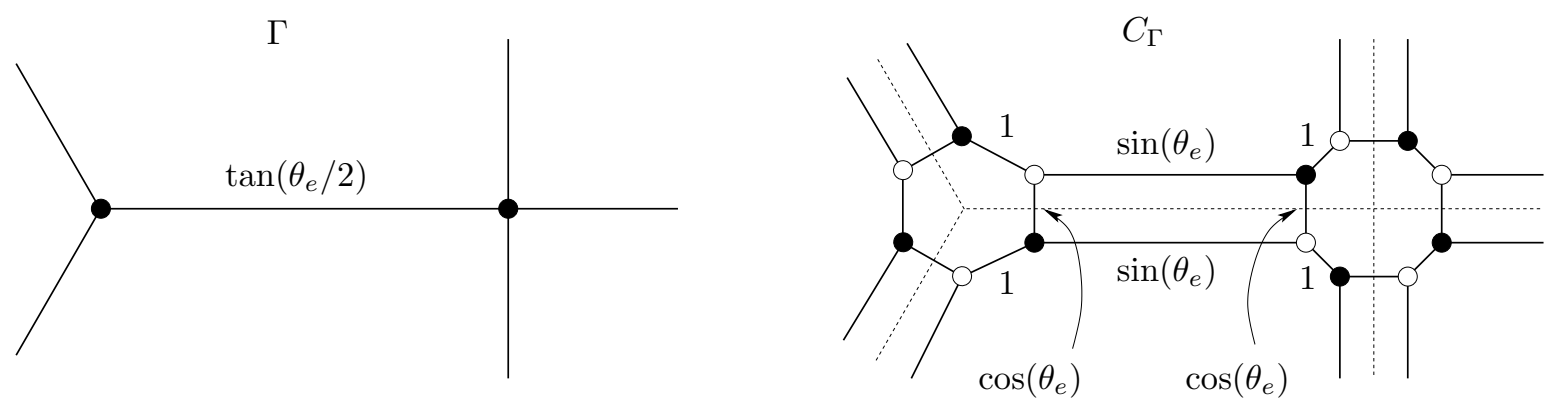

Figure 2. The weighted bipartite graph $C_{\Gamma}$ associated to the weighted graph $\Gamma$.

the oriented edge $e$ counterclockwise. (Since $\Sigma$ is orientable, this can be done in a consistent way.) Note that $(\bar{e})^{*}=\overline{e^{*}}$. As for the dual weights $x^{*} \in[0,1]^{E\left(\Gamma^{*}\right)}$, they are defined via the equality $x+x^{*}+x x^{*}=1$. If we use the parametrization $x=\tan (\theta / 2)$ with $\theta \in[0, \pi / 2]$, then $\theta$ and $\theta^{*}$ are simply related by $\theta+\theta^{*}=\pi / 2$. Note that $\left(\left(\Gamma^{*}\right)^{*},\left(x^{*}\right)^{*}\right)=(\Gamma, x)$.

The double of $(\Gamma, x) \subset \Sigma$ is the weighted bipartite surface graph $(D, y)=\left(D_{\Gamma}, y(x)\right) \subset \Sigma$ given as follows: as a subset of $\Sigma, D_{\Gamma}=\Gamma \cup \Gamma^{*}$, with vertex set $B(D) \cup W(D)$, where $B(D)=V(\Gamma) \cup V\left(\Gamma^{*}\right)=: \Lambda$ and $W(D)=E(\Gamma) \cap E\left(\Gamma^{*}\right)=: \diamond$. As for the weighted edges, an edge $e$ of $\Gamma$ with weight $x_{e}=\tan \left(\theta_{e} / 2\right)$ will give rise to two edges of $D_{\Gamma}$ with weight $\sin \left(\theta_{e}\right)$ and to two transverse edges of $D_{\Gamma}$ with weight $\cos \left(\theta_{e}\right)$. Following [6], we shall denote by $z_{e} \in \diamond$ the vertex of $D_{\Gamma}$ corresponding to the edge $e \in E(\Gamma)$. This is illustrated in Figure 1.

Following Wu-Lin [44] (see also [13, 15), we shall consider another weighted surface graph $(C, y)=\left(C_{\Gamma}, y(x)\right) \subset \Sigma$ associated to a given weighted surface graph $(\Gamma, x) \subset \Sigma$. It is obtained as follows: replace each edge $e$ of $\Gamma$ by a rectangle with the edges parallel to $e$ having weight $\sin \left(\theta_{e}\right)$ while the other two edges have weight $\cos \left(\theta_{e}\right)$. In each corner of each face of $\Gamma \subset \Sigma$, we now have two vertices; join them with an edge of weight 1 . This is illustrated in Figure 2. Note that since the surface $\Sigma$ is orientable, the graph $C_{\Gamma}$ is bipartite. Note also that the weighted graph $\left(C_{\Gamma^{*}}, y\left(x^{*}\right)\right)$ associated to $\left(\Gamma^{*}, x^{*}\right)$ is equal to the weighted graph $\left(C_{\Gamma}, y(x)\right)$ associated to $(\Gamma, x)$.

In our considerations, it will be useful to endow the surface $\Sigma$ with a character of its fundamental group, that is, an element of

$$
\operatorname{Hom}\left(\pi_{1}(\Sigma), \mathbb{C}^{*}\right)=H^{1}\left(\Sigma ; \mathbb{C}^{*}\right) .
$$




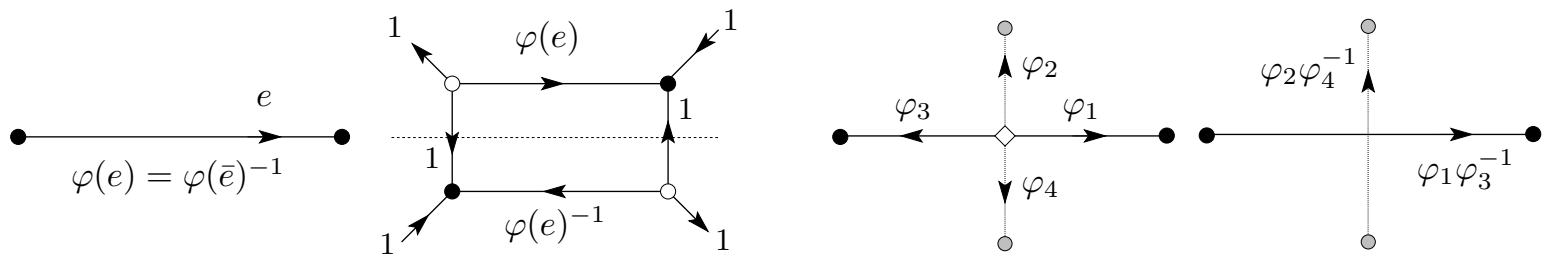

Figure 3. A given cochain $\varphi$ on $\Gamma$, and the associated cochain $\varphi_{C}$ on $C_{\Gamma}$; a cochain $\varphi_{D}$ on $D_{\Gamma}$, and the associated cochains $\varphi$ on $\Gamma$ and $\varphi_{*}$ on $\Gamma^{*}$.

If $\Gamma \subset \Sigma$ is a surface graph, then such a cohomology class can be represented by a 1-cocycle on $\Gamma$. Let us recall that, with the notations above, a 1-cochain is a map $\varphi: \mathbb{E}(\Gamma) \rightarrow \mathbb{C}^{*}$ such that $\varphi(\bar{e})=\varphi(e)^{-1}$ for all $e \in \mathbb{E}(\Gamma)$. It is called a 1-cocycle if for each face $f$ of $\Gamma \subset \Sigma$, $\varphi(\partial f):=\prod_{e \in \partial f} \varphi(e)=1$. Multiplying each $\varphi(e)$ such that $o(e)=v$ by a fixed element of $\mathbb{C}^{*}$ results in another 1-cocycle, which is said to be cohomologous to $\varphi$. Equivalence classes of 1-cocycles define the first cohomology group $H^{1}\left(\Sigma ; \mathbb{C}^{*}\right)$, which only depends on $\Sigma$ (and not on $\Gamma$ ): if $\Sigma$ is a closed surface of genus $g$, then $H^{1}\left(\Sigma ; \mathbb{C}^{*}\right) \simeq\left(\mathbb{C}^{*}\right)^{2 g}$.

If $\Gamma$ is endowed with a 1-cochain $\varphi$, define the associated 1-cochain $\varphi_{C}: \mathbb{E}\left(C_{\Gamma}\right) \rightarrow \mathbb{C}^{*}$ by

$$
\varphi_{C}(w, b)= \begin{cases}\varphi(e) & \text { if }(w, b) \text { runs parallel to } e \in \mathbb{E}(\Gamma) \\ 1 & \text { else, }\end{cases}
$$

as illustrated in Figure 3. Note that if $\varphi$ is a cocycle, then so is $\varphi_{C}$, and they induce the same cohomology class in $H^{1}\left(\Sigma ; \mathbb{C}^{*}\right)$. Also, any 1-cochain $\varphi_{D}$ on $D_{\Gamma}$ naturally induces 1-cochains $\varphi$ on $\Gamma$ and $\varphi_{*}$ on $\Gamma^{*}$, as illustrated in Figure 3. Here again, if $\varphi_{D}$ is a cocycle, then so are $\varphi$ and $\varphi_{*}$, and all three induce the same cohomology class.

2.2. Isoradial graphs. The majority of the results of the present article hold for arbitrary weighted surface graphs, as defined above. However, many of these results take a particularly pleasant form when the graph is isoradially embedded with critical weights. Let us now recall these concepts.

Consider a collection of planar rhombi of equal side length, say $\delta$, each rhombus having a fixed diagonal $e$ and corresponding half-rhombus angle $\theta_{e} \in[0, \pi / 2]$ as illustrated below.

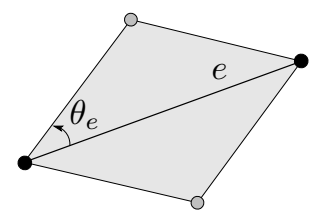

Paste these rhombi together along their sides so that extremities of diagonals are glued to extremities of diagonals. These diagonals then form the edges of a graph $\Gamma$ embedded in a flat surface $\Sigma$, with so-called cone-type singularities in the vertex set $V(\Gamma)$ of $\Gamma$ and in the faces of $\Gamma \subset \Sigma$. The cone angle of the singularity at $v \in V(\Gamma)$ is given by the sum of the angles of the rhombi adjacent to $v$ (and similarly for singularities in the faces).

Definition 1. A graph $\Gamma$ is $\delta$-isoradially embedded in a flat surface $\Sigma$ if it can be realized by pasting rhombi of side length $\delta$ as above, so that all the cone angles are odd multiples of 

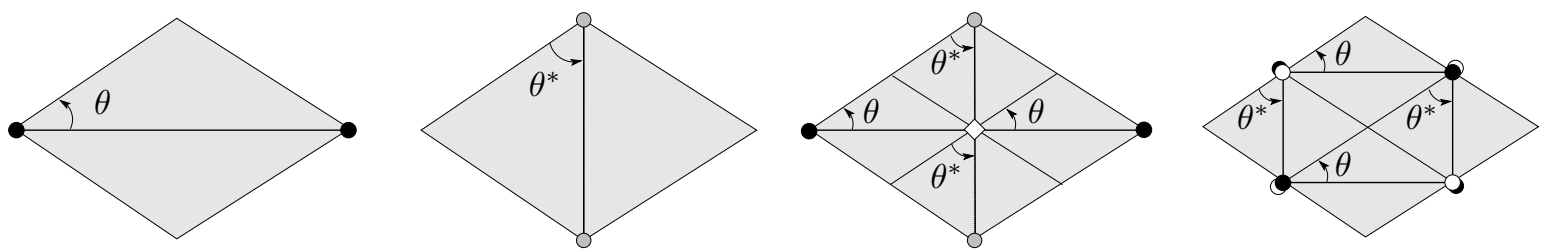

Figure 4 . If $\Gamma$ is isoradially embedded, then so are $\Gamma^{*}, D_{\Gamma}$ and $C_{\Gamma}$.

$2 \pi$. The corresponding critical weights $x=\left(x_{e}\right)_{e \in E(\Gamma)}$ are given by $x_{e}=\tan \left(\theta_{e} / 2\right)$, where $\theta_{e} \in[0, \pi / 2]$ denotes the half-rhombus angle associated to the edge $e \in E(\Gamma)$.

Note that if $\Sigma$ is the (flat) plane, then this definition coincides with the usual notion of isoradial embedding as considered in [27, 6]. It is however more general, as it allows us to work in surfaces of arbitrary genus.

Given a flat surface $\Sigma$ with cone-type singularities, the parallel transport along a closed loop $\gamma$ in $\Sigma$ defines an element of $S O(2)=S^{1}$ called the holonomy of $\gamma$. If all cone-angles are multiples of $2 \pi$, then the holonomy can be described by a homomorphism $\operatorname{Hol} \in \operatorname{Hom}\left(\pi_{1}(\Sigma), S^{1}\right)=$ $H^{1}\left(\Sigma ; S^{1}\right)$. If this homomorphism is trivial, $\Sigma$ is said to have trivial holonomy. (An example of genus $g \geq 1$ is given by the regular $4 g$-gone with opposite sides identified.) If all cone-angles are odd multiples of $2 \pi$, then the inverse square roots of the holonomy can be described by $S^{1}$-valued 1-cocycles on $\Sigma$, which should be thought of as discrete spin structures on $\Sigma$ (see $[$, Section 3.1]).

Note that if $\Gamma$ is $\delta$-isoradially embedded in a flat surface $\Sigma$, then so is the dual graph $\Gamma^{*}$, while the associated bipartite graphs $D_{\Gamma}$ and $C_{\Gamma}$ can be naturally $\frac{1}{2} \delta$-isoradially embedded in this same flat surface $\Sigma$ (some rhombi of $C_{\Gamma}$ being degenerate). Furthermore, if $\Gamma$ is endowed with the critical weights $x$, then the associated weights $x^{*}$ on $\Gamma^{*}$ are nothing but the critical weights determined by the corresponding isoradial embedding. Finally, the associated weights $y$ on $D_{\Gamma}$ and $C_{\Gamma}$ are simply given by $y_{e}=\sin \left(\theta_{e}\right)$, with $\theta_{e}$ the half-rhombus angle determined by the isoradial embedding. This is illustrated in Figure 4 .

2.3. The discrete Laplace operator. Fix a surface graph $\Gamma \subset \Sigma$ endowed with a 1-cocycle $\varphi: \mathbb{E}(\Gamma) \rightarrow \mathbb{C}^{*}$. The space $\mathbb{C}^{V(\Gamma)}$ can be thought of as a combinatorial version of the space of complex valued functions on $\Sigma$, and more generally, of the space of sections of the complex line bundle induced by the class of $\varphi$ in $H^{1}\left(\Sigma ; \mathbb{C}^{*}\right)$. As first observed by Eckmann [17] (in the untwisted case), the Laplace operator on this space can be naturally discretized by an operator $\Delta_{\Gamma}^{\varphi}$ on $\mathbb{C}^{V(\Gamma)}$, provided one fixes inner products on the cochain spaces $C^{0}(\Gamma ; \mathbb{C})$ and $C^{1}(\Gamma ; \mathbb{C})$. Furthermore, if these inner products are diagonal (i.e. given by positive vertex weights $\left\{\mu_{v}\right\}_{v \in V(\Gamma)}$ and edge weights $\left.\left\{\nu_{e}\right\}_{e \in E(\Gamma)}\right)$, then this operator takes the following very simple form (see e.g. [19]): for $f \in \mathbb{C}^{V(\Gamma)}$ and $v \in V(\Gamma)$,

$$
\left(\Delta_{\Gamma}^{\varphi} f\right)(v)=\frac{1}{\mu_{v}} \sum_{e=(v, w)} \nu_{e}(f(v)-f(w) \varphi(e)) .
$$

Finally, if the surface $\Sigma$ is endowed with a Riemannian metric, then a sensible choice for these weights consists in taking for $\mu_{v}$ the area of the star of $v$ in $\Gamma$, and for $\nu_{e}$ the quotient of the 
length of $e^{*}$ over the length of $e$. With the isoradial case in mind, this leads to the following definition.

Definition 2. Let $(\Gamma, x) \subset \Sigma$ be a weighted surface graph, with weights parametrized by $x_{e}=\tan \left(\theta_{e} / 2\right)$. The associated discrete Laplace operator is the operator $\Delta_{\Gamma}^{\varphi}$ on $\mathbb{C}^{V(\Gamma)}$ defined by

$$
\left(\Delta_{\Gamma}^{\varphi} f\right)(v)=\frac{1}{\mu_{v}} \sum_{e=(v, w)} \tan \left(\theta_{e}\right)(f(v)-f(w) \varphi(e))
$$

for $f \in \mathbb{C}^{V(\Gamma)}$ and $v \in V(\Gamma)$, where the sum is over all oriented edges of the form $e=(v, w)$, and $\mu_{v}=\frac{1}{2} \sum_{e \in \mathbb{E}(\Gamma)_{v}} \sin \left(2 \theta_{e}\right)$.

We refer to the recent paper [28] for a beautiful example of the relevance of this operator in statistical mechanics.

2.4. The Kac-Ward operator. To define the next operator, one needs to be able to measure rotation angles along curves. For planar closed curves, there is a unique sensible way to do so: one measures the rotation angle of the velocity vector field of the curve with respect to any constant vector field on the plane. For curves embedded in an arbitrary surface, this is more tricky. As it turns out, there is a standard geometrical tool to do this, which is called a spin structure. We shall not go into the trouble of recalling their formal definition (see e.g. [1, p.55]); let us only mention that such a spin structure can be given by a vector field on $\Sigma$ with isolated zeroes of even index. Let us also recall that the group of orientationpreserving diffeomorphisms of $\Sigma$ acting on the set of spin structures on $\Sigma$ defines two orbits: the even spin structures - with so-called Arf invariant equal to 0 -, and the odd ones, with Arf invariant equal to 1.

So, given a weighted surface graph $(\Gamma, x) \subset \Sigma$, let us endow $\Sigma$ with a Riemannian metric and let us fix a vector field $\lambda$ on $\Sigma$ with isolated zeroes of even index in $\Sigma \backslash \Gamma$. Also, fix a 1-cochain $\varphi: \mathbb{E}(\Gamma) \rightarrow \mathbb{C}^{*}$.

Definition 3. The associated Kac-Ward operator is the operator $K W^{\varphi}=K W^{\varphi}(\Gamma, x)$ on $\mathbb{C}^{\mathbb{E}(\Gamma)}$ defined by

$$
\left(K W^{\varphi} f\right)(e)=f(e)-\varphi(e) x_{e} \sum_{\substack{e^{\prime} \in \mathbb{E}(\Gamma) v \\ e^{\prime} \neq \bar{e}}} \exp \left(\frac{i}{2} \alpha_{\lambda}\left(e, e^{\prime}\right)\right) f\left(e^{\prime}\right)
$$

for $f \in \mathbb{C}^{\mathbb{E}(\Gamma)}$ and $e \in \mathbb{E}(\Gamma)$ with $t(e)=v$, the sum being over all $e^{\prime} \in \mathbb{E}(\Gamma)$ starting where $e$ finishes, but different from $\bar{e}$. Here, $\alpha_{\lambda}\left(e, e^{\prime}\right)$ is the rotation angle (in radians) of the velocity vector field along $e$ followed by $e^{\prime}$ with respect to the vector field $\lambda$, from $z_{e}$ to $z_{e^{\prime}}$. (See Figure 5.)

If the cocycle is trivial, we shall simply denote this operator by $K W(\Gamma, x)$, or by $K W^{\lambda}(\Gamma, x)$ if we wish to underline its dependence on the spin structure $\lambda$.

The relevance of this operator for the study of the Ising model is given by the following facts [7. It is well-known that the space $H^{1}(\Sigma ;\{ \pm 1\})$ acts freely transitively on the set of spin structures on $\Sigma$. Now, for any 1-cocycle $\varphi: \mathbb{E}(\Gamma) \rightarrow\{ \pm 1\}$, the determinant of the matrix $K W^{\varphi}(\Gamma, x)$ is the square of a polynomial in the variables $\left\{x_{e}\right\}_{e \in E(\Gamma)}$ which only depends on the spin structure determined by the action of the class of $\varphi$ on $\lambda$, and on the surface graph 


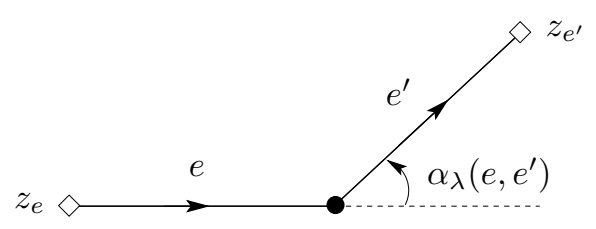

Figure 5. The angle $\alpha_{\lambda}\left(e, e^{\prime}\right)$ in the planar case.

$\Gamma \subset \Sigma$ (see subsection 4.3 for more details). Finally, if $\operatorname{det}\left(K W^{\varphi}(\Gamma, x)\right)^{1 / 2}$ denotes the square root with constant coefficient equal to +1 , then the high temperature expansion of the Ising partition function on $\Gamma$ is given by

$$
Z^{I s i n g}(\Gamma, x)=\frac{1}{2^{g}} \sum_{\varphi \in H^{1}(\Sigma ;\{ \pm 1\})}(-1)^{\operatorname{Arf}(\varphi)} \operatorname{det}\left(K W^{\varphi}(\Gamma, x)\right)^{1 / 2},
$$

where $g$ is the genus of $\Sigma$ and $\operatorname{Arf}(\varphi) \in \mathbb{Z}_{2}$ is the Arf invariant of the spin structure obtained by the action of $\varphi$ on $\lambda$. Note that in the planar case, this equality simply reads $Z^{\text {Ising }}(\Gamma, x)=$ $\operatorname{det}(K W(\Gamma, x))^{1 / 2}$; this is the original Kac-Ward formula [23, 14].

2.5. The Kasteleyn operator. Recall that a Kasteleyn orientation [24, 25, 26] on a bipartite surface graph $G \subset \Sigma$ can be understood as a map $\omega: E(G) \rightarrow\{ \pm 1\}$ such that for each face $f$ of $G$,

$$
\omega(\partial f):=\prod_{e \in \partial f} \omega(e)=(-1)^{\frac{|\partial f|}{2}+1} .
$$

Two Kasteleyn orientations are called equivalent if they can be related by flipping the orientation of all edges adjacent to a finite number of vertices.

So, fix a bipartite weighted graph $(G, y) \subset \Sigma$ with vertex set $V(G)=B \cup W$, a 1-cochain $\varphi: \mathbb{E}(G) \rightarrow \mathbb{C}^{*}$, and a Kasteleyn orientation $\omega$ on $G \subset \Sigma$.

Definition 4. The associated Kasteleyn operator is the operator $K^{\varphi}=K^{\varphi}(G, y): \mathbb{C}^{B} \rightarrow \mathbb{C}^{W}$ defined by

$$
\left(K^{\varphi} f\right)(w)=\sum_{e=(w, b)} \varphi(e) \omega(e) y_{e} f(b)
$$

for $f \in \mathbb{C}^{B}$ and $w \in W$, the sum being over all oriented edges of $G$ of the form $e=(w, b)$.

If the cocycle is trivial, we shall simply denote this operator by $K(G, y)$, or by $K^{\omega}(G, y)$ if we wish to underline its dependence on the Kasteleyn orientation $\omega$.

The relevance of this operator for the study of the dimer model is given by the following formula [41, 20, 11]. The space $H^{1}(\Sigma ;\{ \pm 1\})$ acts freely transitively on the set of equivalence classes of Kasteleyn orientations on $G \subset \Sigma$, and this set is in equivariant correspondence with the set of spin structures on $\Sigma$ [11. Then, the partition function of the dimer model on $G$ is given by the formula

$$
Z^{\text {dimer }}(G, y)=\frac{1}{2^{g}} \sum_{\varphi \in H^{1}(\Sigma ;\{ \pm 1\})}(-1)^{\operatorname{Arf}(\varphi)} \operatorname{det}\left(K^{\varphi}(G, y)\right)
$$


where $\operatorname{Arf}(\varphi) \in \mathbb{Z}_{2}$ denotes the Arf invariant of the spin structure obtained by the action of $\varphi$ on the spin structure corresponding to $\omega$. Note that in the planar case, this equality simply reads $Z^{\text {dimer }}(G, y)=\operatorname{det}(K(G, y))$; this is Kasteleyn's celebrated theorem [26].

Note that since the spaces $\mathbb{C}^{B}$ and $\mathbb{C}^{W}$ are not identical, the determinant of the Kasteleyn operators is a priori not well-defined. However, we will only be interested in the case where the number of white and black vertices of $G$ are equal. (Otherwise, both sides of the equation displayed above vanish.) In this case, the determinants are well-defined up to a global sign. Since they also change sign when the Kasteleyn orientation is replaced by an equivalent one, the formula above should really be understood as holding up to a global sign, or for a good choice of a Kasteleyn orientation. (One can also simply use absolute values on the right hand side.)

2.6. The discrete Dirac operator. Dirac operators can be defined on surfaces endowed with a complex structure and with a hermitian metric. Let us therefore consider a bipartite surface graph $G \subset \Sigma$ isoradially embedded in a flat surface $\Sigma$, together with a fixed nowhere vanishing vector field $X$ at the vertices of $G$. (See [9, Section 2.1] for a motivation of these assumptions.) Given an oriented edge $e=\left(v, v^{\prime}\right)$ of $G$, we shall write $\vartheta_{X}(e)$ for the oriented angle between $X(v)$ and $e$, as illustrated below. Finally, let us write $\mu_{v}=\frac{1}{2} \sum_{e \in \mathbb{E}(G)_{v}} \sin \left(2 \theta_{e}\right)$, and fix a 1-cochain $\varphi: \mathbb{E}(G) \rightarrow \mathbb{C}^{*}$.

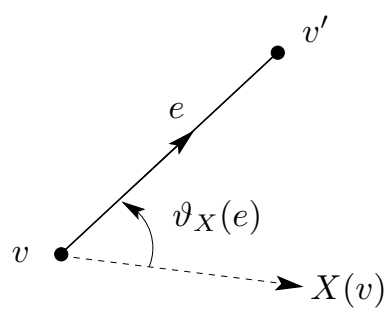

Definition 5. The associated discrete $\bar{\partial}$-operator is the operator $\bar{\partial}^{\varphi}=\bar{\partial}_{G}^{\varphi}: \mathbb{C}^{B} \rightarrow \mathbb{C}^{W}$ defined by

$$
\left(\bar{\partial}^{\varphi} f\right)(w)=\frac{1}{\mu_{w}} \sum_{e=(w, b)} \varphi(e) \exp \left(i \vartheta_{X}(e)\right) \sin \left(\theta_{e}\right) f(b)
$$

for $f \in \mathbb{C}^{B}$ and $w \in W$, while the discrete $\partial$-operator $\partial^{\varphi}=\partial_{G}^{\varphi}: \mathbb{C}^{W} \rightarrow \mathbb{C}^{B}$ is defined by

$$
\left(\partial^{\varphi} f\right)(b)=\frac{1}{\mu_{b}} \sum_{e=(b, w)} \varphi(e) \exp \left(-i \vartheta_{X}(e)\right) \sin \left(\theta_{e}\right) f(w)
$$

for $f \in \mathbb{C}^{W}$ and $b \in B$. Finally the associated discrete Dirac operator is the operator $\not D^{\varphi}=\not D_{G}^{\varphi}=\left(\begin{array}{cc}0 & -\partial^{\varphi} \\ \bar{\partial}^{\varphi} & 0\end{array}\right): \mathbb{C}^{B} \oplus \mathbb{C}^{W} \rightarrow \mathbb{C}^{B} \oplus \mathbb{C}^{W}$.

Note that if $\Sigma$ is the flat plane (with $\varphi=1$ and $X$ a constant vector field), then these definitions coincide with the ones of [27, 6]. More generally, if $G$ is isoradially embedded in a flat surface and $\varphi$ is a discrete spin structure, then we get back 9, Definition 3.9]. It should come as no surprise that a vector field is required: on Riemann surfaces, the $\partial$ and $\bar{\partial}$ operators take values in $(1,0)$ and $(0,1)$-forms, respectively. In any case, a function $f \in \mathbb{C}^{B}$ being discrete holomorphic at $w \in W$, i.e. satisfying $\left(\bar{\partial}^{\varphi} f\right)(w)=0$, is independent of the vector field. 


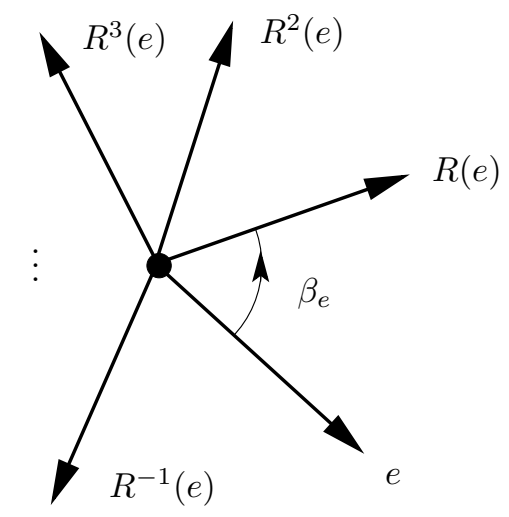

Figure 6. The rotated edges, and the angle $\beta_{e}$.

We will be particularly interested in two families of bipartite graphs, namely the graphs $D_{\Gamma}$ and $C_{\Gamma}$ associated to an arbitrary isoradial graph $\Gamma$ as described in subsection 2.1. The corresponding Dirac operators will be denoted by $\not_{D}^{\varphi}=\left(\begin{array}{cc}0 & -\partial_{D}^{\varphi} \\ \bar{\partial}_{D}^{\varphi} & 0\end{array}\right)$ and $\not_{C}^{\varphi}=\left(\begin{array}{cc}0 & -\partial_{C}^{\varphi} \\ \bar{\partial}_{C}^{\varphi} & 0\end{array}\right)$.

\section{RELATiOnS BETWEEN OPERATORS}

The aim of this section is to exhibit natural relations between the operators defined in Section 2, A couple of applications are also included, but the most interesting consequences will be presented in Section 4. More precisely, we begin by showing one of our main results: the fact that the Kac-Ward operator on an arbitrary surface graph $\Gamma$ and the Kasteleyn operator on the associated bipartite graph $C_{\Gamma}$ can be explicitly related (subsection 3.1). As an immediate consequence, we obtain a Kramers-Wannier type duality result for Kac-Ward determinants. In subsection 3.2, we show that in the isoradial case, the Kasteleyn and discrete Dirac operators on $C_{\Gamma}$ are conjugate. In the next paragraph, we observe that the square of the Dirac operator on $C_{\Gamma}$ is closely related to the discrete Laplace operator on an associated graph. Finally, in subsection 3.4, we relate the discrete Dirac operators on $C_{\Gamma}$ and on the double $D_{\Gamma}$ of $\Gamma$. Note that the results of these last two subsections will not be referred to in the rest of the paper.

3.1. Kac-Ward versus Kasteleyn operators. In this paragraph, we relate the Kac-Ward operator on an arbitrary weighted surface graph $(\Gamma, x) \subset \Sigma$ to the Kasteleyn operator on the associated bipartite graph $\left(C_{\Gamma}, y(x)\right) \subset \Sigma$ (Theorem 3.1). As an immediate consequence, we obtain an equality between their respective determinants (Corollary 3.2). Note that such a Kac-Ward determinant is known to be equal to the Kasteleyn determinant of the associated Fisher graph (see [7, Proposition 4.6]). However, Fisher graphs are never bipartite, thus not allowing to use the full power of the dimer model theory. In the present case, not only is $C_{\Gamma}$ bipartite, but Corollary 3.2 immediately implies a generalized Kramers-Wannier duality for Kac-Ward determinants on arbitrary weighted surface graphs (Corollaries 3.3 and 3.4). These statements simultaneously generalize [8, Theorem 4.4], which deals with the critical isoradial case, and [10, Corollaries 3.3 and 3.4], which deal with the toric case. 


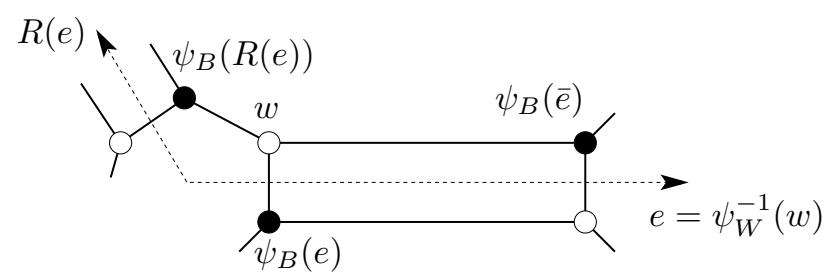

Figure 7 . The bijections $\psi_{B}: \mathbb{E} \rightarrow B$ and $\psi_{W}: \mathbb{E} \rightarrow W$.

To state the main result of this section, we need to introduce several notations. Let $(\Gamma, x) \subset \Sigma$ be an arbitrary weighted surface graph, $\Sigma$ being endowed with a Riemannian metric, and assume that the edges of $\Gamma$ are smoothly embedded in $\Sigma$. Also, let us fix a vector field $\lambda$ on $\Sigma$ with zeroes of even index in $\Sigma \backslash \Gamma$. For any $e \in \mathbb{E}(\Gamma)=$ : $\mathbb{E}$, write $D_{e}=\exp \left(i a_{e}\right)$, where $a_{e}$ denotes the oriented angle at $z_{e}$ between $\lambda$ and $e$. (The letters $D$ and $a$ stand for "direction" and "argument", respectively.) Note the equality $D_{\bar{e}}=-D_{e}$. Given a vertex $v \in V(\Gamma)$, let us cyclically order the elements of $\mathbb{E}_{v}$ by turning counterclockwise around $v$. (As $\Sigma$ is orientable, this can be done in a consistent way.) For $e \in \mathbb{E}_{v}$, let $R(e) \in \mathbb{E}_{v}$ denote the next edge with respect to this cyclic order, and set $\beta_{e}=\pi+\alpha_{\lambda}(\bar{e}, R(e))$. By definition of $\alpha_{\lambda}$ (recall Definition 3), $\beta_{e}$ is the rotation angle between $e$ and $R(e)$ measured with respect to the fixed vector field $\lambda$ (see Figure 6). Note the equality $D_{R(e)}=\exp \left(i \beta_{e}\right) D_{e}$. Finally, set $q_{e}=\exp \left(\frac{i}{2} \beta_{e}\right)$.

This allows us to define automorphisms $J, R, D$ and $q$ of the space $\mathbb{C}^{\mathbb{E}}: J$ is simply given by $(J f)(e)=f(\bar{e})$, while $R$ is defined by $(R f)(e)=f(R(e))$. Finally, we shall write $D$ for the diagonal automorphism of $\mathbb{C}^{\mathbb{E}}$ given by $(D f)(e)=D_{e} f(e)$, and similarly for $q$, for any weight system, and for $\varphi$. To conclude this long list of notations, let $\psi_{B}: \mathbb{E} \rightarrow B:=B\left(C_{\Gamma}\right)$ (resp. $\psi_{W}: \mathbb{E} \rightarrow W:=W\left(C_{\Gamma}\right)$ ) denote the bijection mapping each oriented edge $e$ of $\Gamma$ to the unique black (resp. white) vertex of $C_{\Gamma}$ immediately to the right (resp. left) of $e$, as illustrated in Figure 7. These bijections induce isomorphisms $\psi_{B}: \mathbb{C}^{B} \rightarrow \mathbb{C}^{\mathbb{E}}$ and $\psi_{W}: \mathbb{C}^{W} \rightarrow \mathbb{C}^{\mathbb{E}}$.

Theorem 3.1. For any 1-cochain $\varphi: \mathbb{E} \rightarrow \mathbb{C}^{*}$, the following diagram commutes:

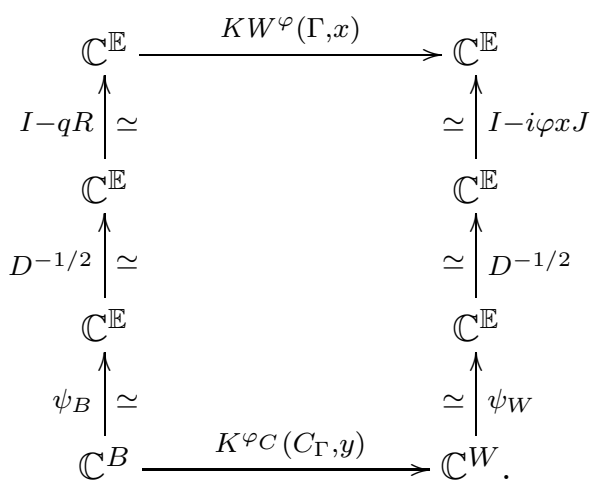

Before proving this theorem, let us make a couple of remarks. First, note that the choice of the square root of the operator $D$ has no importance: changing the sign of the square root of the coefficient $D_{e}$ (say, on the left-hand side of the diagram) simply amounts to inverting the Kasteleyn orientation of all edges adjacent to $\psi_{B}(e)$, i.e. replacing it with an equivalent Kasteleyn orientation. Also, the dependence of this diagram on the equivalence class of the 
Kasteleyn orientation is well-understood. Such equivalence classes are in natural one-toone correspondence with spin structures on $\Sigma$ (see [11, Section 4]), which can be described by vector fields with zeroes of even index. Moreover, the paper [11 contains an explicit construction of such a vector field $\lambda_{\omega}$ from a Kasteleyn orientation $\omega$, and one can check that the diagram above commutes for such an orientation $\omega$ and vector field $\lambda_{\omega}$.

Proof of Theorem 3.1. We shall start with the most technical part of the demonstration, i.e. the computation of the composition $(I-i \varphi x J)^{-1} K W^{\varphi}(I-q R)$. To achieve this goal, it will be convenient to consider the operator $\operatorname{Succ} \in \operatorname{End}\left(\mathbb{C}^{\mathbb{E}}\right)$ defined as follows: if $e$ is an oriented edge with terminus $t(e)=v$, then

$$
\operatorname{Succ}(f)(e)=\varphi(e) x_{e} \sum_{e^{\prime} \in \mathbb{E}_{v}} \omega\left(e, e^{\prime}\right) f\left(e^{\prime}\right),
$$

where $\omega\left(e, e^{\prime}\right)=\exp \left(\frac{i}{2} \alpha_{\lambda}\left(e, e^{\prime}\right)\right)$ for $e^{\prime} \neq \bar{e} \in \mathbb{E}_{v}$ and $\omega(e, \bar{e})=-i$. Also, let $T \in \operatorname{End}\left(\mathbb{C}^{\mathbb{E}}\right)$ be the endomorphism given by $T=S u c c+i \varphi x J$. By definition, the Kac-Ward operator $K W^{\varphi}$ is equal to $I-T$. Now, the operator $(I+i \varphi x J)(I-T)$ decomposes into

$$
(I+i \varphi x J)(I-T)=I-S u c c+C o m,
$$

where

$$
\operatorname{Com}(f)(e)=(-i \varphi x J T)(f)(e)=-i \varphi(e) x_{e}(T f)(\bar{e})=-i x_{e}^{2} \sum_{\substack{e^{\prime} \in \mathbb{E} v \\ e^{\prime} \neq e}} \omega\left(\bar{e}, e^{\prime}\right) f\left(e^{\prime}\right)
$$

if $e$ has origin $o(e)=v$. Let us compute the composition $(I+i \varphi x J)(I-T)(I-q R)$ using the decomposition displayed above. If $e$ has terminus $t(e)=v$, then

$$
\begin{aligned}
(\operatorname{Succ}(I-q R))(f)(e) & =\operatorname{Succ}(f)(e)-\operatorname{Succ}(q(R(f)))(e) \\
& =\varphi(e) x_{e} \sum_{e^{\prime} \in \mathbb{E}_{v}} \omega\left(e, e^{\prime}\right)\left(f\left(e^{\prime}\right)-q_{e^{\prime}} f\left(R\left(e^{\prime}\right)\right)\right) \\
& =\varphi(e) x_{e} \sum_{e^{\prime} \in \mathbb{E}_{v}}\left(\omega\left(e, e^{\prime}\right)-\omega\left(e, R^{-1}\left(e^{\prime}\right)\right) q_{R^{-1}\left(e^{\prime}\right)}\right) f\left(e^{\prime}\right) \\
& =-2 i \varphi(e) x_{e} f(\bar{e}) .
\end{aligned}
$$

Therefore, we have the equality $\operatorname{Succ}(I-q R)=-2 i \varphi x J$. Similarly, given $e$ with origin $o(e)=v$,

$$
\begin{aligned}
(\operatorname{Com}(I-q R))(f)(e)= & \operatorname{Com}(f)(e)-\operatorname{Com}(q(R(f)))(e) \\
= & -i x_{e}^{2} \sum_{e^{\prime} \in \mathbb{E}_{v} \backslash\{e\}} \omega\left(\bar{e}, e^{\prime}\right)\left(f\left(e^{\prime}\right)-q_{e^{\prime}} f\left(R\left(e^{\prime}\right)\right)\right) \\
= & -i x_{e}^{2} \sum_{e^{\prime} \in \mathbb{E}_{v} \backslash\{e, R(e)\}}\left(\omega\left(\bar{e}, e^{\prime}\right)-\omega\left(\bar{e}, R^{-1}\left(e^{\prime}\right)\right) q_{R^{-1}\left(e^{\prime}\right)}\right) f\left(e^{\prime}\right) \\
& +i x_{e}^{2}\left(\omega\left(\bar{e}, R^{-1}(e)\right) q_{R^{-1}(e)} f(e)-\omega(\bar{e}, R(e)) f(R(e))\right) \\
= & -x_{e}^{2}(I+q R)(f)(e) .
\end{aligned}
$$


These two equalities lead to

$$
\begin{aligned}
(I+i \varphi x J)(I-T)(I-q R) & =(I-S u c c+\operatorname{Com})(I-q R) \\
& =(I-q R)+2 i \varphi x J-x^{2}(I+q R) \\
& =\left(1-x^{2}\right)+2 x i \varphi J-\left(1+x^{2}\right) q R .
\end{aligned}
$$

This allows us to determine the operator $M^{\varphi}$ defined by the commuting diagram

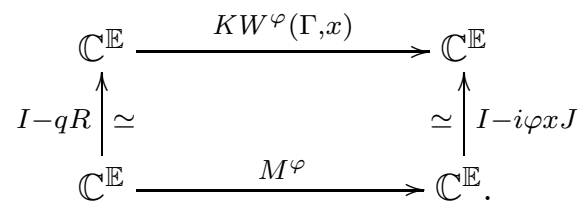

It is given by

$$
\begin{aligned}
M^{\varphi} & =(I-i \varphi x J)^{-1}(I-T)(I-q R) \\
& =\frac{1}{1+x^{2}}(I+i \varphi x J)(I-T)(I-q R) \\
& =\frac{1-x^{2}}{1+x^{2}}+\frac{2 x}{1+x^{2}} i \varphi J-q R \\
& =\cos (\theta)+\sin (\theta) i \varphi J-q R,
\end{aligned}
$$

using the parametrization $x=\tan (\theta / 2)$ of the weights.

Now, observe that the three maps $\psi_{B} \circ \psi_{W}^{-1}, \psi_{B} \circ{ }^{-} \circ \psi_{W}^{-1}$ and $\psi_{B} \circ R \circ \psi_{W}^{-1}$ associate to a fixed $w \in W$ the three black vertices of $C_{\Gamma}$ adjacent to $w$, as demonstrated in Figure 7 . Therefore, the operator $\widetilde{K}^{\varphi}$ defined by the commuting diagram

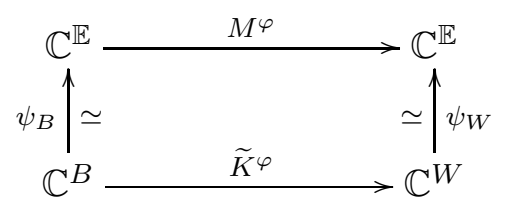

is given by the coefficients

$$
\widetilde{K}_{w b}^{\varphi}= \begin{cases}\cos \left(\theta_{e}\right) & \text { if the edge }(w, b) \text { is perpendicular to } e \in \mathbb{E} ; \\ \varphi(e) i \sin \left(\theta_{e}\right) & \text { if }(w, b) \text { is to the left of } e \in \mathbb{E} ; \\ -q_{e} & \text { if }(w, b) \text { is in the "corner" of } e \text { and } R(e) ; \\ 0 & \text { if } w \text { and } b \text { are not adjacent in } C_{\Gamma} .\end{cases}
$$

This is illustrated below.

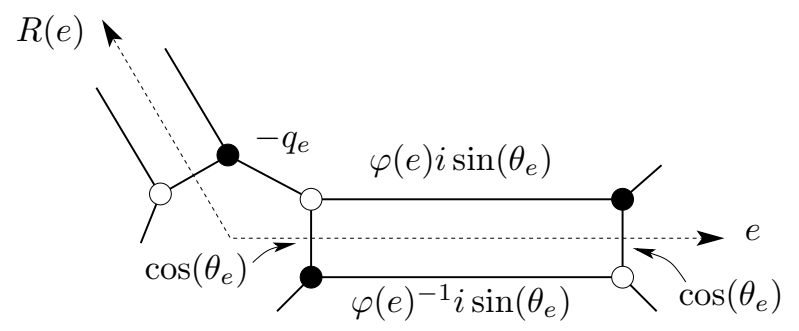


In other words, $\widetilde{K}^{\varphi}$ is precisely the Kasteleyn operator of $\left(C_{\Gamma}, y\right)$ associated to the 1-cocycle $\varphi_{C}: \mathbb{E}\left(C_{\Gamma}\right) \rightarrow \mathbb{C}^{*}$ (recall Figure 3) and to the map $\tilde{\omega}: E\left(C_{\Gamma}\right) \rightarrow S^{1} \subset \mathbb{C}^{*}$ given by

$$
\tilde{\omega}(w, b)= \begin{cases}1 & \text { if }(w, b) \text { is perpendicular to } e \in \mathbb{E} ; \\ i & \text { if }(w, b) \text { is to the left of } e \in \mathbb{E} ; \\ -q_{e} & \text { if }(w, b) \text { is in the corner of } e \text { and } R(e) .\end{cases}
$$

Extend this map to a 1-cochain $\tilde{\omega}: \mathbb{E}\left(C_{\Gamma}\right) \rightarrow S^{1}$ by setting $\tilde{\omega}(b, w)=\tilde{\omega}(w, b)^{-1}$. Now, observe that for any face $f$ of $C_{\Gamma} \subset \Sigma$,

$$
\tilde{\omega}(\partial f)=\prod_{e \in \partial f} \tilde{\omega}(e)=(-1)^{\frac{|\partial f|}{2}+1} .
$$

This is obvious for the rectangular faces; for the faces corresponding to vertices of $\Gamma$, use the fact that the angles $\beta_{e}$ add up to $2 \pi$ around each vertex; for the faces corresponding to faces of $\Gamma$, use the fact that the angles $\alpha_{\lambda}\left(e, e^{\prime}\right)$ add up to an odd multiple of $2 \pi$ around each face since the vector field $\lambda$ has zeroes of even index. Furthermore, if $\gamma$ denotes a 1-cycle in $C_{\Gamma} \subset \Sigma$, then one easily checks that $\tilde{\omega}(\gamma)^{2}=\exp \left(i \operatorname{rot}_{\lambda}(\gamma)\right)=1$, where $\operatorname{rot}_{\lambda}(\gamma)$ is the rotation angle of the velocity vector field along the closed curve $\gamma$ with respect to $\lambda$. Therefore, the $S^{1}$-valued 1-cochain $\tilde{\omega}$ is cohomologous to a Kasteleyn orientation $\omega$. In other words, $\tilde{\omega}$ can be transformed into $\omega$ by a sequence of the following transformation: multiply all the edges adjacent to a fixed vertex of $C_{\Gamma}$ by some complex number of modulus 1 . This defines diagonal operators $d_{B}$ and $d_{W}$ such that the following diagram commutes:

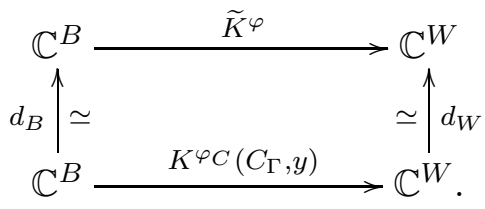

By connectedness of $\Gamma$, these diagonal operators are unique up to simultaneous multiplication by a fixed element of $S^{1}$.

To complete the proof of the theorem, it remains to check that

$$
\psi_{B} \circ d_{B}=D^{-1 / 2} \circ \psi_{B} \quad \text { and } \quad \psi_{W} \circ d_{W}=D^{-1 / 2} \circ \psi_{W}
$$

for some well-chosen square root of the operator $D$. To do so, let us make the diagonal operators $d_{B}=\left(d_{b}\right)_{b \in B}$ and $d_{W}=\left(d_{w}\right)_{w \in W}$ more explicit. Focusing on the rectangular face of $C_{\Gamma}$ around the edge $e$ leads to the equations $d_{\psi_{W}(e)}= \pm d_{\psi_{B}(e)}$ and $d_{\psi_{W}(e)}= \pm i d_{\psi_{B}(\bar{e})}$. Similarly, looking at the face of $C_{\Gamma}$ around the vertex $v$ leads to the equations $d_{\psi_{B}(R(e))}=$ $\pm q_{e}^{-1} d_{\psi_{W}(e)}$ for all $e \in \mathbb{E}_{v}$. In a nutshell, the operators $d_{B}^{2}$ and $d_{W}^{2}$ are uniquely determined (up to a global rotation) by the equations

$$
d_{\psi_{B}(e)}^{2}=d_{\psi_{W}(e)}^{2}=-d_{\psi_{B}(\bar{e})}^{2} \quad \text { and } \quad d_{\psi_{B}(R(e))}^{2}=\exp \left(-i \beta_{e}\right) d_{\psi_{B}(e)}^{2} \quad \text { for all } e \in \mathbb{E} .
$$

By definition, the operator $D$ satisfies the equations $D_{e}=-D_{\bar{e}}$ and $D_{R(e)}=\exp \left(i \beta_{e}\right) D_{e}$. It follows that $d_{B}, d_{W}$ can be chosen so that $d_{\psi_{B}(e)}^{2}=d_{\psi_{W}(e)}^{2}=D_{e}^{-1}$ for all $e \in \mathbb{E}$. This is equivalent to the equalities $\psi_{\bullet} \circ d_{\bullet}=D^{-1 / 2} \circ \psi_{\bullet}$ for $\bullet=B, W$, and the proof is completed. 
Theorem 3.1 easily leads to the following result, which was first obtained in the genus one case by Duminil-Copin and the author [10]. (On how to make sense of the determinant of the Kasteleyn operator, recall the remark at the end of paragraph 2.5.)

Corollary 3.2. Given a weighted surface graph $(\Gamma, x) \subset \Sigma$, there is a Kasteleyn orientation on $C_{\Gamma} \subset \Sigma$ such that

$$
\operatorname{det}\left(K W^{\varphi}(\Gamma, x)\right)=2^{-|V(\Gamma)|} \prod_{e \in E(\Gamma)}\left(1+x_{e}^{2}\right) \operatorname{det}\left(K^{\varphi_{C}}\left(C_{\Gamma}, y\right)\right)
$$

for any 1-cochain $\varphi: \mathbb{E} \rightarrow \mathbb{C}^{*}$.

Proof. First note that

$$
\operatorname{det}(I-i \varphi x J)=\prod_{e \in E(\Gamma)} \operatorname{det}\left(\begin{array}{cc}
1 & -i \varphi(\bar{e}) x_{e} \\
-i \varphi(e) x_{e} & 1
\end{array}\right)=\prod_{e \in E(\Gamma)}\left(1+x_{e}^{2}\right) .
$$

Also, the partition $\mathbb{E}=\bigsqcup_{v \in V(\Gamma)} \mathbb{E}_{v}$ induces a decomposition $I-q R=\bigoplus_{v \in V(\Gamma)}(I-q R)_{v}$. This leads to

$$
\operatorname{det}(I-q R)=\prod_{v \in V(\Gamma)} \operatorname{det}\left((I-q R)_{v}\right)=\prod_{v \in V(\Gamma)}\left(1-\prod_{e \in \mathbb{E}_{v}} q_{e}\right)=2^{|V(\Gamma)|},
$$

since $\sum_{e \in \mathbb{E}_{v}} \beta_{e}=2 \pi$. Hence, Theorem 3.1 implies that the equality of the corollary holds for any 1-cochain $\varphi$ up to multiplication by a fixed complex number of modulus 1. For $\varphi$ taking values in $\{ \pm 1\}$, both sides are real; therefore, the equality holds up to a global sign. One can then choose a Kasteleyn orientation such that the identity holds.

Let us now assume that $\varphi: \mathbb{E} \rightarrow \mathbb{C}^{*}$ is a 1-cocycle. In this case, one easily checks that the determinant of $K W^{\varphi}(\Gamma, x)$ only depends on the cohomology class of $\varphi$ in $H^{1}\left(\Sigma ; \mathbb{C}^{*}\right)$. By abuse of notation, we shall also denote such a class by $\varphi$. Here is the announced generalized Kramers-Wannier duality.

Corollary 3.3. For any weighted graph $(\Gamma, x) \subset \Sigma$ and any class $\varphi \in H^{1}\left(\Sigma ; \mathbb{C}^{*}\right)$,

$$
2^{|V(\Gamma)|} \prod_{e \in E(\Gamma)}\left(1+x_{e}\right)^{-1} \operatorname{det}\left(K W^{\varphi}(\Gamma, x)\right)=2^{\left|V\left(\Gamma^{*}\right)\right|} \prod_{e \in E(\Gamma)}\left(1+x_{e}^{*}\right)^{-1} \operatorname{det}\left(K W^{\varphi}\left(\Gamma^{*}, x^{*}\right)\right) .
$$

Proof. As mentioned in subsection 2.1, the weighted graph $\left(C_{\Gamma^{*}}, y\left(x^{*}\right)\right)$ associated to $\left(\Gamma^{*}, x^{*}\right)$ is equal to the weighted graph $\left(C_{\Gamma}, y(x)\right)$ associated to $(\Gamma, x)$. Fix a cocycle $\varphi_{D}$ on $D_{\Gamma}$ as in Figure 3 with $\varphi_{1} \varphi_{3}^{-1}=\varphi_{2} \varphi_{4}^{-1}$ - any class can be represented by such a cocycle - and consider the associated cocycles $\varphi, \varphi_{*}, \varphi_{C}$ and $\left(\varphi_{*}\right)_{C}$, who all define the same cohomology class. The cocycles $\varphi_{C}$ and $\left(\varphi_{*}\right)_{C}$ are not equal, but cohomologous, and the corresponding transformations of the Kasteleyn matrices do not change their determinant. Corollary 3.2 together with the equality $\frac{1+x^{2}}{1+x}=\frac{1+\left(x^{*}\right)^{2}}{1+x^{*}}$ then gives the result.

As mentioned in subsection 2.4, for a cocycle $\varphi: \mathbb{E}(\Gamma) \rightarrow\{ \pm 1\} \operatorname{det}\left(K W^{\varphi}(\Gamma, x)\right)$ is the square of a polynomial in the weight variables $x_{e}$. As the constant coefficient of this determinant is equal to 1 , we can pick such a square root $\left|K W^{\varphi}(\Gamma, x)\right|^{1 / 2}$ by requiring its constant coefficient to be +1 . Taking a closer look at the sign leads to the following duality. 
Corollary 3.4. For any weighted graph $(\Gamma, x) \subset \Sigma$ and any $\varphi \in H^{1}(\Sigma ;\{ \pm 1\})$,

$$
\left.2^{\frac{|V(\Gamma)|}{2}} \prod_{e \in E(\Gamma)}\left(1+x_{e}\right)^{-\frac{1}{2}}\left|K W^{\varphi}(\Gamma, x)^{\frac{1}{2}}=(-1)^{A(\varphi)} 2^{\frac{\left|V\left(\Gamma^{*}\right)\right|}{2}} \prod_{e \in E(\Gamma)}\left(1+x_{e}^{*}\right)^{-\frac{1}{2}}\right| K W^{\varphi}\left(\Gamma^{*}, x^{*}\right)\right|^{\frac{1}{2}},
$$

where $A(\varphi) \in \mathbb{Z}_{2}$ is the Arf invariant of the spin structure obtained by the action of $\varphi$ on $\lambda$.

Proof. By Corollary 3.3, we only need to determine the sign $A(\varphi)$ in the equation above. Let $g$ denote the genus of $\Sigma$. Setting $x=1$ (and therefore, $x^{*}=0$ ) leads to

$$
\left|K W^{\varphi}(\Gamma, 1)\right|^{1 / 2}=(-1)^{A(\varphi)} 2^{\left(\left|V\left(\Gamma^{*}\right)\right|+|E(\Gamma)|-|V(\Gamma)|\right) / 2}=(-1)^{A(\varphi)} 2^{\left|V\left(\Gamma^{*}\right)\right|+g-1},
$$

using the fact that $|V(G)|-|E(G)|+\left|V\left(G^{*}\right)\right|$ is equal to the Euler characteristic $\chi(\Sigma)=2-2 g$. Furthermore, the Ising partition function $Z^{I s i n g}(\Gamma, x)$ with weights $x=1$ is nothing but the cardinality of the $\mathbb{Z}_{2}$-vector space of 1 -cycles modulo 2 in $\Gamma$. Since $\Gamma$ is connected, the dimension of this space is classically equal to $|E(\Gamma)|-|V(\Gamma)|+1$. The Kac-Ward formula (1) now implies

$$
2^{|E(\Gamma)|-|V(\Gamma)|+1}=Z^{I s i n g}(\Gamma, 1)=\frac{1}{2^{g}} \sum_{\varphi \in H^{1}(\Sigma ;\{ \pm 1\})}(-1)^{\operatorname{Arf}(\varphi)}(-1)^{A(\varphi)} 2^{\left|V\left(G^{*}\right)\right|+g-1},
$$

that is,

$$
\sum_{\varphi \in H^{1}(\Sigma ;\{ \pm 1\})}(-1)^{\operatorname{Arf}(\varphi)+A(\varphi)}=2^{2-\chi(\Sigma)}=2^{2 g} .
$$

Since the set $H^{1}(\Sigma ;\{ \pm 1\})$ has precisely $2^{2 g}$ elements, this shows that $A(\varphi)=\operatorname{Arf}(\varphi)$ for all $\varphi$, and the corollary is proved.

To illustrate the power of Theorem 3.1, let us finally show how it allows us to easily transfer the recent results Kenyon-Sun-Wilson [30] from the dimer to the Ising model. Consider a locally finite planar graph $\mathscr{G}$ with edge weights $J=\left(J_{e}\right)_{e} \in(0, \infty)^{E(\mathscr{G})}$ and faces that are bounded topological discs. Let us assume that this weighted graph is biperiodic, i.e. invariant under the action of a lattice $L \simeq \mathbb{Z}^{2}$. For $n \geq 1$, let $\Gamma_{n}$ denote the finite weighted toric graph given by $\Gamma_{n}=\mathscr{G} / n L \subset \mathbb{T}^{2}$, and set $\Gamma_{1}=: \Gamma$. Fixing a basis of $L$, one can identify $H^{1}\left(\mathbb{T}^{2} ; \mathbb{C}^{*}\right)$ with $\left(\mathbb{C}^{*}\right)^{2}$ so that any 1 -cocycle $\varphi: \mathbb{E} \rightarrow \mathbb{C}^{*}$ defines a pair $(z, w)$ of non-vanishing complex numbers. Finally, let us write $P_{n}(z, w)=\operatorname{det} K W^{\varphi}\left(\Gamma_{n}, x\right)$, where $x_{e}=\tanh \left(\beta J_{e}\right)$, set $P_{1}=: P$, and denote by $H=\left(\begin{array}{cc}A_{z} & B \\ B & A_{w}\end{array}\right)$ the Hessian of $P$ at $(1,1)$.

Corollary 3.5. At the critical inverse temperature $\beta=\beta_{c}$, the Ising partition function on $\Gamma_{n}$ satisfies

$$
\log Z_{\beta_{c}}^{J}\left(\Gamma_{n}\right)=n^{2} f\left(\beta_{c}\right)+\mathrm{fsc}_{1}(\tau)+o(1),
$$

where $f$ is the free energy per fundamental domain

$$
f(\beta)=|V(\Gamma)| \log (2)+\sum_{e \in E(\Gamma)} \log \cosh \left(\beta J_{e}\right)+\frac{1}{2(2 \pi i)^{2}} \int_{\mathbb{T}^{2}} \log P(z, w) \frac{d z}{z} \frac{d w}{w},
$$

$\tau$ is the modular parameter given by

$$
\tau=\frac{-B+i \sqrt{A_{z} A_{w}-B^{2}}}{A_{w}},
$$

and $\operatorname{fsc}_{1}(\tau)$ is the explicit universal finite-size correction term given in [30, Theorem 2 (a)], which is invariant under modular transformations. 
Proof. Given a cocycle $\varphi: \mathbb{E}(\Gamma) \rightarrow \mathbb{C}^{*}$ representing a class $(z, w) \in\left(\mathbb{C}^{*}\right)^{2}$, let us write $Q(z, w)=\operatorname{det} K^{\varphi_{C}}\left(C_{\Gamma}, y\right)$. (Recall that the cocycles $\varphi$ and $\varphi_{C}$ define the same cohomology class.) Corollary 3.2 now reads

$$
P(z, w)=2^{-|V(\Gamma)|} \prod_{e \in E(\Gamma)}\left(1+x_{e}^{2}\right) Q(z, w),
$$

and implies that the curves defined by $P(z, w)$ and $Q(z, w)$ coincide. Since $C_{\Gamma}$ is bipartite, one can apply results of Kenyon-Okounkov-Sheffield [29] and conclude that this is a special Harnack curve. As demonstrated in [10, it allows us to show that $P(z, w)$ is strictly positive on $\mathbb{T}^{2} \backslash\{(1,1)\}$, and vanishes at $(1,1)$ if and only if the inverse temperature is critical. Recall that the high temperature expansion 43 for the Ising model on $\left(\Gamma_{n}, J\right)$ can be stated as

$$
Z_{\beta}^{J}\left(\Gamma_{n}\right)=2^{n^{2}|V(\Gamma)|}\left(\prod_{e \in E(\Gamma)} \cosh \left(\beta J_{e}\right)\right)^{n^{2}} Z^{\text {Ising }}\left(\Gamma_{n}, x\right),
$$

while the genus one Kac-Ward formula (1) reads

$$
Z^{I s i n g}\left(\Gamma_{n}, x\right)=\frac{1}{2}\left(-P_{n}(1,1)^{1 / 2}+P_{n}(-1,1)^{1 / 2}+P_{n}(1,-1)^{1 / 2}+P_{n}(-1,-1)^{1 / 2}\right) .
$$

The conclusion now follows from [30, Theorem 1] in the special case $E=\left(\begin{array}{cc}n & 0 \\ 0 & n\end{array}\right)$, applied to the equation above.

Example. Consider the case of the rectangular lattice $\mathscr{G}$ with horizontal (resp. vertical) edges having weight $J$ (resp. $K$ ). Fixing the natural fundamental domain and basis of $\mathbb{Z}^{2}$, we obtain

$$
P(z, w)=\left(1+x^{2}\right)\left(1+y^{2}\right)-x\left(1-y^{2}\right)\left(z+z^{-1}\right)-y\left(1-x^{2}\right)\left(w+w^{-1}\right),
$$

where $x=\tanh (\beta J)$ and $y=\tanh (\beta K)$. This leads to

$$
H=\left(\begin{array}{cc}
-2 x\left(1-y^{2}\right) & 0 \\
0 & -2 y\left(1-x^{2}\right)
\end{array}\right) \text { and } \tau=i \sqrt{\frac{y\left(1-x^{2}\right)}{x\left(1-y^{2}\right)}} .
$$

Now, the model is at the critical temperature if and only if $x$ and $y$ satisfy the equality $1=x+y+x y$. Using the parametrization $x=\tan (\theta / 2)$ and $y=\tan (\rho / 2)$, this equation can be written as $\theta+\rho=\frac{\pi}{2}$. This leads to the modular parameter $\tau=i \tan (\theta)$, which defines the isoradial embedding of $\mathscr{G}$ whose critical weights are the ones we started with.

3.2. Kasteleyn versus discrete Dirac operators. Let us now assume that the graph $\Gamma$ is isoradially embedded in a flat surface $\Sigma$, and endowed with the corresponding critical weights $x_{e}=\tan \left(\theta_{e} / 2\right)$ (recall paragraph 2.2). Then, the associated graph $C_{\Gamma}$ is also isoradially embedded in $\Sigma$, so one can define a discrete $\bar{\partial}$-operator $\bar{\partial}_{C}^{\varphi}: \mathbb{C}^{B} \rightarrow \mathbb{C}^{W}$ as explained in paragraph 2.6. Recall that this discretization is particularly relevant when the 1-cochain $\varphi$ is a so-called discrete spin structure, that is, when it represents one of the $2^{2 g}$ inverse square roots of the holonomy of the flat metric on $\Sigma$. Recall also that this operator $\bar{\partial}_{C}^{\varphi}$ depends on the choice of a vector field $X$ at the white vertices of $C_{\Gamma}$; without loss of generality, we shall assume that this vector field at the vertex $w=\psi_{W}(e)$ points towards $\psi_{B}(e) \in B$ (recall Figure 7).

The main result of this paragraph is that the Kasteleyn operators associated to $2^{2 g}$ nonequivalent Kasteleyn orientations on $\left(C_{\Gamma}, y\right)$ are simultaneously conjugate to the discrete 
$\bar{\partial}$-operators associated to $2^{2 g}$ non-cohomologous discrete spin structures. This can be understood as an instructive and explicit special case of [9, Theorem 4.8], which deals with general bipartite isoradial graphs. (See also [27, Theorem 10.1] for the planar case).

Proposition 3.6. Given any Kasteleyn orientation $\omega$ on $C_{\Gamma} \subset \Sigma$, there exists a unique discrete spin structure $\varphi_{\omega}: \mathbb{E}\left(C_{\Gamma}\right) \rightarrow S^{1}$ such that the following diagram commutes

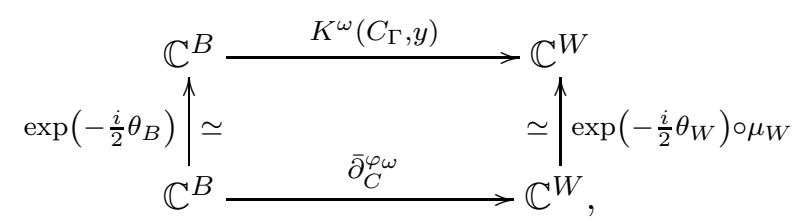

where the diagonal operators $\theta_{B}=\left(\theta_{b}\right)_{b \in B}, \theta_{W}=\left(\theta_{w}\right)_{w \in B}$ and $\mu_{W}=\left(\mu_{w}\right)_{w \in W}$ are defined by $\theta_{b}=\theta_{e}$ if $b=\psi_{B}(e), \theta_{w}=\theta_{e}$ if $w=\psi_{W}(e)$, and $\mu_{w}=\frac{1}{2} \sum_{e \in \mathbb{E}\left(C_{\Gamma}\right)_{w}} \sin \left(2 \theta_{e}\right)$. Furthermore, the map $\omega \mapsto \varphi_{\omega}$ defines a bijection between equivalence classes of Kasteleyn orientations on $C_{\Gamma} \subset \Sigma$ and inverse square roots in $H^{1}\left(\Sigma ; S^{1}\right)$ of the holonomy on $\Sigma$.

Proof. Fix $f \in \mathbb{C}^{B}$ and $w \in W$. Following the notations of Figure 7 , let us write $e=\psi_{W}^{-1}(w)$, $e^{\prime}=R(e)$, and $b_{1}=\psi_{B}(e), b_{2}=\psi_{B}(\bar{e}), b_{3}=\psi_{B}\left(e^{\prime}\right)$ for the three vertices of $C_{\Gamma}$ adjacent to $w$. By definition, we have

$$
\begin{aligned}
\left(K^{\omega}\left(C_{\Gamma}, y\right) \circ \exp \left(-\frac{i}{2} \theta_{B}\right)\right) & (f)(w)=\omega\left(w, b_{1}\right) \exp \left(-\frac{i}{2} \theta_{e}\right) \cos \left(\theta_{e}\right) f\left(b_{1}\right)+ \\
& +\omega\left(w, b_{2}\right) \exp \left(-\frac{i}{2} \theta_{e}\right) \sin \left(\theta_{e}\right) f\left(b_{2}\right)+\omega\left(w, b_{3}\right) \exp \left(-\frac{i}{2} \theta_{e^{\prime}}\right) f\left(b_{3}\right),
\end{aligned}
$$

and

$$
\begin{aligned}
&\left(\exp \left(-\frac{i}{2} \theta_{W}\right) \circ \mu_{W} \circ \bar{\partial}_{C}^{\varphi}\right)(f)(w)=\exp \left(-\frac{i}{2} \theta_{e}\right)\left(\varphi\left(w, b_{1}\right) \cos \left(\theta_{e}\right) f\left(b_{1}\right)+\right. \\
&\left.+\varphi\left(w, b_{2}\right) i \sin \left(\theta_{e}\right) f\left(b_{2}\right)-\varphi\left(w, b_{3}\right) \exp \left(i \theta_{e}\right) f\left(b_{3}\right)\right) .
\end{aligned}
$$

Therefore, the diagram commutes if and only if the 1-cochain $\varphi: \mathbb{E}\left(C_{\Gamma}\right) \rightarrow S^{1}$ is given by

$$
\varphi\left(w, b_{j}\right)= \begin{cases}\omega\left(w, b_{1}\right) & \text { if } j=1 \\ -i \omega\left(w, b_{2}\right) & \text { if } j=2 \\ -\exp \left(-\frac{i}{2}\left(\theta_{e}+\theta_{e^{\prime}}\right)\right) \omega\left(w, b_{3}\right) & \text { if } j=3\end{cases}
$$

Using the fact that $\omega$ is a Kasteleyn orientation and the fact that the cone angles of the singularities of $\Sigma$ are odd multiples of $2 \pi$, one checks that $\varphi$ is a 1-cocycle. Furthermore, if $\gamma$ is any closed curve in $C_{\Gamma}, \varphi(\gamma)^{-2}$ is nothing but the holonomy $\operatorname{Hol}(\gamma)$. Therefore, $\varphi$ is a discrete spin structure. The last statement follows easily from the definitions.

3.3. Dirac versus Laplace operators. In this whole paragraph, we shall assume that $\Gamma$ is a graph isoradially embedded in a flat surface $\Sigma$ with trivial holonomy. On such a surface, one can fix a constant vector field $X$, which we will be using to evaluate the associated discrete Dirac operators (recall Definition 5).

The following relation is well-known in the planar 27] and toric 2] cases. The proof is straightforward. 
Proposition 3.7. Given any graph $\Gamma$ isoradially embedded in a flat surface with trivial holonomy and any 1-cocycle $\varphi_{D}: \mathbb{E}\left(D_{\Gamma}\right) \rightarrow \mathbb{C}^{*}$,

$$
-\partial_{D}^{\varphi_{D}} \circ \bar{\partial}_{D}^{\varphi_{D}}=\Delta_{\Gamma}^{\varphi} \oplus \Delta_{\Gamma^{*}}^{\varphi_{*}}
$$

as endomorphisms of $\mathbb{C}^{B(D)}=\mathbb{C}^{V(\Gamma)} \oplus \mathbb{C}^{V\left(\Gamma^{*}\right)}$.

Note that the composition $-\bar{\partial}_{D}^{\varphi_{D}} \circ \partial_{D}^{\varphi_{D}}$ does not seem to be related to the discrete Laplace operator in any sensible way. Therefore, it is not clear how to make sense of the square of the discrete Dirac operator $\left(\not D_{D}^{\varphi}\right)^{2}=\left(\begin{array}{cc}0 & -\partial_{D}^{\varphi} \\ \bar{\partial}_{D}^{\varphi} & 0\end{array}\right)^{2}=\left(\begin{array}{cc}-\partial_{D}^{\varphi} \bar{\partial}_{D}^{\varphi} & 0 \\ 0 & -\bar{\partial}_{D}^{\varphi} \partial_{D}^{\varphi}\end{array}\right)$.

On the other hand, considering the bipartite graph $C_{\Gamma}$ instead of $D_{\Gamma}$ leads to a more symmetrical result, as we now explain. First, note that in the smooth setting, a direct computation leads to the equality $\not D^{2}=\left(\begin{array}{cc}-\partial \bar{\partial} & 0 \\ 0 & -\bar{\partial} \partial\end{array}\right)=\left(\begin{array}{cc}\Delta-i A & 0 \\ 0 & \Delta+i A\end{array}\right)$, where $A=\frac{\partial^{2}}{\partial x \partial y}-\frac{\partial^{2}}{\partial y \partial x}$ vanishes. As we will see, this is reflected in the discretization by $C_{\Gamma}$.

Given an isoradially embedded graph $\Gamma$, let $M$ denote the (isoradial) graph dual to $D_{\Gamma}$, as illustrated below. Also, let $\varepsilon$ denote the orientation of the edges of $M$ illustrated in the same figure.

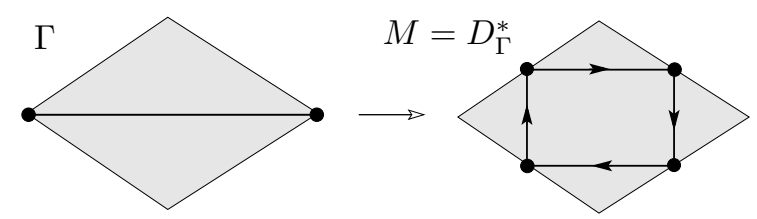

Note that $C_{\Gamma}$ can be obtained from $M$ by replacing each vertex by a "small" edge between two vertices. (This operation is very natural in the setting of discrete complex analysis, see 9 , Section 2.2].) Without loss of generality, we shall consider 1-cochains $\varphi$ on $C_{\Gamma}$ that are trivial on the small edges of $C_{\Gamma}$, and use the same notation $\varphi$ for the restriction to the edges of $M$. Finally, we shall denote by $A_{\varepsilon}^{\varphi}$ the associated normalized $\varphi$-twisted skew-adjacency operator on $\mathbb{C}^{V(M)}$. In other "words",

$$
\left(A_{\varepsilon}^{\varphi} f\right)(v)=\frac{1}{\mu_{v}} \sum_{e=\left(v, v^{\prime}\right)} \varepsilon(e) \varphi(e) f\left(v^{\prime}\right)
$$

for $f \in \mathbb{C}^{V(M)}$ and $v \in V(M)$, the sum being over all edges of $M$ of the form $\left(v, v^{\prime}\right)$, with $\varepsilon(e)=1$ if $\varepsilon$ orients $e$ from $v$ to $v^{\prime}$, and $\varepsilon(e)=-1$ else.

Proposition 3.8. Given any graph $\Gamma$ isoradially embedded in a flat surface with trivial holonomy and any 1-cocycle $\varphi: \mathbb{E}\left(C_{\Gamma}\right) \rightarrow \mathbb{C}^{*}$ which is trivial on the small edges of $C_{\Gamma}$,

$$
\left(\not D_{C}^{\varphi}\right)^{2}=\left(\begin{array}{cc}
-\partial_{C}^{\varphi} \bar{\partial}_{C}^{\varphi} & 0 \\
0 & -\bar{\partial}_{C}^{\varphi} \partial_{C}^{\varphi}
\end{array}\right)=\frac{1}{2}\left(\begin{array}{cc}
\Delta_{M}^{\varphi}-i A_{\varepsilon}^{\varphi} & 0 \\
0 & \Delta_{M}^{\varphi}+i A_{\varepsilon}^{\varphi}
\end{array}\right)
$$

as endomorphisms of $\mathbb{C}^{V(C)}=\mathbb{C}^{V(M)} \oplus \mathbb{C}^{V(M)}$. In particular,

$$
-\left(\partial_{C}^{\varphi} \bar{\partial}_{C}^{\varphi}+\bar{\partial}_{C}^{\varphi} \partial_{C}^{\varphi}\right)=\Delta_{M}^{\varphi} .
$$


Proof. By definition, for any $b \in B:=B\left(C_{\Gamma}\right)$ and $f \in \mathbb{C}^{B}$,

$$
-\left(\partial_{C}^{\varphi} \circ \bar{\partial}_{C}^{\varphi}\right)(f)(b)=\frac{-1}{2 \mu_{b}} \sum_{e=(b, w)} \sum_{e^{\prime}=\left(w, b^{\prime}\right)} \varphi(e) \varphi\left(e^{\prime}\right) \frac{\sin \left(\theta_{e}\right) \sin \left(\theta_{e^{\prime}}\right)}{\sin \left(\theta_{w}\right) \cos \left(\theta_{w}\right)} \exp \left(i\left(\vartheta\left(e^{\prime}\right)-\vartheta(e)\right)\right) f\left(b^{\prime}\right),
$$

where $\theta_{w}$ stands for $\theta_{\psi_{W}^{-1}(w)}$ and $\vartheta$ for $\vartheta_{X}$. Let us denote the vertices of $C_{\Gamma}$ around $b$ as illustrated below.

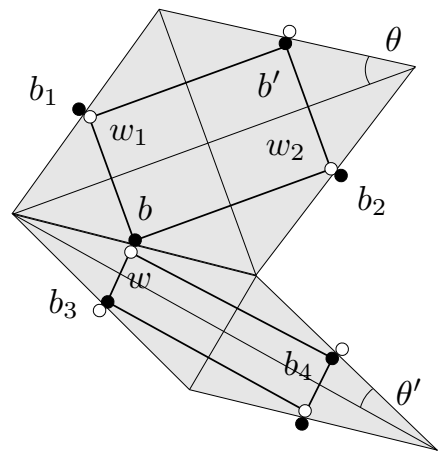

Also, we shall denote by $v$ (resp. $v_{1}, v_{2}$ ) the vertex of $M$ corresponding to $b$ and $w$ (resp. $b_{1}$ and $w_{1}, b_{2}$ and $\left.w_{2}\right)$. Since $\varphi$ is a cochain and $\vartheta(\bar{e})-\vartheta(e)= \pm \pi$, the coefficient of $f(b)$ in the formula above is given by

$$
\frac{1}{2}\left(\tan (\theta)+\tan \left(\theta^{*}\right)+\tan \left(\theta^{\prime}\right)+\tan \left(\left(\theta^{\prime}\right)^{*}\right)\right),
$$

where $\theta^{*}=\frac{\pi}{2}-\theta$ as usual. This coincides with the coefficient of $f(v)$ in $\frac{1}{2} \Delta_{M}^{\varphi}(f)(v)$. As for the coefficient of $f\left(b^{\prime}\right)$, since $\varphi$ is a cocycle and $\vartheta\left(w_{2}, b^{\prime}\right)-\vartheta\left(b, w_{2}\right)=\frac{\pi}{2}$ while $\vartheta\left(w_{1}, b^{\prime}\right)-\vartheta\left(b, w_{1}\right)=$ $-\frac{\pi}{2}$, it vanishes as expected. Since $\varphi$ is trivial on the small edges and $\vartheta\left(w_{1}, b_{1}\right)-\vartheta\left(b, w_{1}\right)=\theta$, the coefficient of $f\left(b_{1}\right)$ is equal to

$$
\frac{-1}{2 \mu_{b}} \varphi\left(b, w_{1}\right) \frac{\cos (\theta)}{\cos (\theta) \sin (\theta)} \exp (i \theta)=\frac{-1}{2 \mu_{b}} \varphi\left(v, v_{1}\right)\left(\tan \left(\theta^{*}\right)+i\right)
$$

which coincides with the coefficient of $f\left(v_{1}\right)$ in $\frac{1}{2}\left(\Delta_{M}^{\varphi}-i A_{\varepsilon}^{\varphi}\right)(f)(v)$. Similarly, the coefficient of $f\left(b_{2}\right)$ is equal to

$$
\frac{-1}{2 \mu_{b}} \varphi\left(b, w_{2}\right) \frac{\sin (\theta)}{\cos (\theta) \sin (\theta)} \exp \left(-i \theta^{*}\right)=\frac{-1}{2 \mu_{b}} \varphi\left(v, v_{2}\right)(\tan (\theta)-i),
$$

which is the coefficient of $f\left(v_{2}\right)$ in $\frac{1}{2}\left(\Delta_{M}^{\varphi}-i A_{\varepsilon}^{\varphi}\right)(f)(v)$. The cases of $f\left(b_{3}\right)$ and $f\left(b_{4}\right)$ are treated similarly, leading to the equation

$$
-\partial_{C}^{\varphi} \bar{\partial}_{C}^{\varphi}=\frac{1}{2}\left(\Delta_{M}^{\varphi}-i A_{\varepsilon}^{\varphi}\right) .
$$

The formula for $-\bar{\partial}_{C}^{\varphi} \partial_{C}^{\varphi}$ follows, since the coefficients of $\bar{\partial}_{C}$ and $\partial_{C}$ are complex conjugate. 


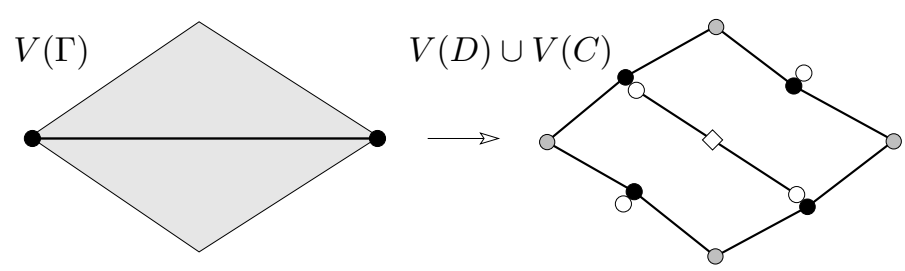

3.4. Comparing discrete Dirac operators. Surprisingly, the discrete Dirac operators $\not_{D}^{\varphi}$ on $D=D_{\Gamma}$ and $\not_{C}^{\varphi}$ on $C=C_{\Gamma}$ are also related in a fairly natural way. To present this result, it is convenient to adopt the following terminology: we will say that vertices $v \in V(D)=\Lambda \cup \diamond$ and $v^{\prime} \in V(C)=B \cup W$ are adjacent, denoted $v \sim v^{\prime}$, if they are linked by an edge in the right-hand side of the illustration below.

Clearly, any 1-cocycle $\varphi$ on the graph above naturally induces 1-cocycles $\varphi_{D}$ on $D$ and $\varphi_{C}$ on $C$. We shall denote by $h_{D C}^{\varphi}: \mathbb{C}^{V(D)} \rightarrow \mathbb{C}^{V(C)}$ and $h_{C D}^{\varphi}: \mathbb{C}^{V(C)} \rightarrow \mathbb{C}^{V(D)}$ the associated twisted adjacency operators, i.e. the operators defined by

$$
\left(h_{D C}^{\varphi} f\right)\left(v^{\prime}\right)=\frac{\operatorname{deg}\left(v^{\prime}\right)}{2} \sum_{v^{\prime} \sim v \in V(D)} \varphi\left(v^{\prime}, v\right) f(v) \quad \text { and } \quad\left(h_{C D}^{\varphi} g\right)(v)=\sum_{v \sim v^{\prime} \in V(C)} \varphi\left(v, v^{\prime}\right) g\left(v^{\prime}\right)
$$

for $f \in \mathbb{C}^{V(D)}, g \in \mathbb{C}^{V(C)}, v \in V(D)$ and $v^{\prime} \in V(C)$, where $\operatorname{deg}\left(v^{\prime}\right) \in\{1,2\}$ denotes the number of vertices in $V(D)$ adjacent to $v^{\prime}$.

Proposition 3.9. Given any graph $\Gamma$ isoradially embedded in a flat surface with trivial holonomy and any 1-cocycle $\varphi$ as above, the following diagram commutes:

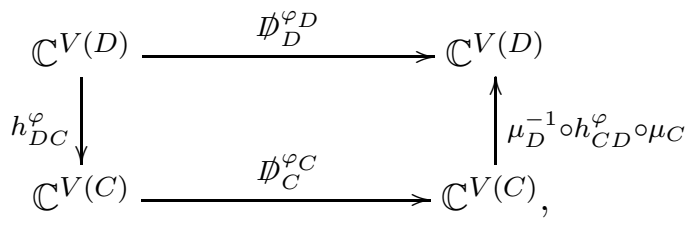

where $\mu_{D}=\left(\mu_{v}\right)_{v \in V(D)}$ and $\mu_{C}=\left(\mu_{v^{\prime}}\right)_{v^{\prime} \in V(C)}$ are the usual diagonal operators.

Proof. Let $f$ be an element of $\mathbb{C}^{V(D)}$. Fixing $z \in \diamond \subset V(D)$, let us denote by $\left(v_{1}, v_{2}, v_{3}, v_{4}\right)$ the vertices of $\Lambda$ around $z$ numbered counterclockwise, and by $\theta$ the corresponding half-rhombus angle (assuming $v_{1}$ and $v_{3}$ are vertices of the primal graph $\Gamma$ ). By definition, we have

$$
\begin{aligned}
\left(h_{C D}^{\varphi} \circ \mu_{C} \circ \not_{C}^{\varphi_{C}} \circ h_{D C}^{\varphi}\right)(f)(z) & =\sum_{z \sim w \in W} \sum_{(w, b) \in E(C)} \sum_{b \sim v \in \Lambda} \varphi_{D}(z, v) e^{i \vartheta_{X}(w, b)} \sin \left(\theta_{w b}\right) f(v) \\
& =e^{i \vartheta_{X}\left(v_{3}, v_{1}\right)} \sum_{j} \varphi_{D}\left(z, v_{j}\right) c_{j} f\left(v_{j}\right),
\end{aligned}
$$

where the coefficients $c_{j}$ are given by $c_{1}=i \cos (\theta)+e^{-i \theta^{*}}=\sin (\theta), c_{2}=\sin (\theta)+i e^{i \theta}=i \cos (\theta)$, $c_{3}=-i \cos (\theta)+i e^{i \theta}=-\sin (\theta)$ and $c_{4}=-\sin (\theta)+e^{-i \theta^{*}}=-i \cos (\theta)$. Therefore,

$$
\left(h_{C D}^{\varphi} \circ \mu_{C} \circ \not D_{C}^{\varphi_{C}} \circ h_{D C}^{\varphi}\right)(f)(z)=\left(\mu_{D} \circ \bar{\partial}_{D}^{\varphi_{D}}\right)(f)(z)=\left(\mu_{D} \circ \not D_{D}^{\varphi_{D}}\right)(f)(z) .
$$


Furthermore, fixing $v \in \Lambda$, we have

$$
\begin{aligned}
\left(h_{C D}^{\varphi} \circ \mu_{C} \circ \not_{C}^{\varphi_{C}} \circ h_{D C}^{\varphi}\right) & (f)(v)=-\frac{1}{2} \sum_{v \sim b \in B} \sum_{(b, w) \in E(C)} \sum_{w \sim z \in \diamond} \varphi_{D}(v, z) e^{-i \vartheta_{X}(b, w)} \sin \left(\theta_{b w}\right) f(z) \\
& =-\frac{1}{2} \sum_{(v, z) \in E(D)} \varphi_{D}(v, z) e^{-i \vartheta_{X}(v, z)}\left(\sin \left(\theta_{z}\right)+i \cos \left(\theta_{z}\right)+e^{-i \theta_{z}^{*}}\right) f(z) \\
& =-\sum_{(v, z) \in E(D)} \varphi_{D}(v, z) e^{-i \vartheta_{X}(v, z)} \sin \left(\theta_{z}\right) f(z) \\
& =-\left(\mu_{D} \circ \bar{\partial}_{D}^{\varphi_{D}}\right)(f)(v) \\
& =\left(\mu_{D} \circ \not D_{D}^{\varphi_{D}}\right)(f)(v),
\end{aligned}
$$

and the proof is completed.

Let us conclude this section with one last summarizing remark. Given any isoradially embedded graph $\Gamma \subset \Sigma$ with critical weights $x$, Corollary 3.2 and Proposition [3.6 give the relation between determinants

$$
\left|K W^{\varphi}(\Gamma, x)\right|^{2} \doteq\left|K^{\varphi}\left(C_{\Gamma}, y(x)\right)\right|^{2} \doteq\left|\bar{\partial}_{C}^{\varphi}\right|^{2},
$$

where $\doteq$ stands for the equality between functions of $\varphi \in H^{1}\left(\Sigma ; \mathbb{C}^{*}\right) \simeq\left(\mathbb{C}^{*}\right)^{2 g}$ up to a multiplicative constant. Furthermore, if $\varphi$ is unitary, i.e. belongs to $\left(S^{1}\right)^{2 g}$, then $\bar{\partial}_{D}^{\varphi}$ and $\partial_{D}^{\varphi}$ are adjoint matrices, which are square if $g=1$. Assuming that $\Sigma$ is a torus, Proposition 3.7 therefore leads to

$$
\left|\bar{\partial}_{D}^{\varphi}\right|^{2}=\left|\partial_{D}^{\varphi} \circ \bar{\partial}_{D}^{\varphi}\right| \doteq\left|\Delta_{\Gamma}^{\varphi} \oplus \Delta_{\Gamma^{*}}^{\varphi}\right| \doteq\left|\Delta_{\Gamma}^{\varphi}\right|^{2} .
$$

(The last equality is well-known, see e.g. [2].) Finally, Proposition 3.9 seems to hint at a relation of the form $\left|\bar{\partial}_{C}^{\varphi}\right| \doteq\left|\bar{\partial}_{D}^{\varphi}\right|$, which would imply the equality $\left|K W^{\varphi}(\Gamma, x)\right| \doteq\left|\Delta_{\Gamma}^{\varphi}\right|$. This equality actually holds under the conditions stated above, and only under these conditions, as was proved in [8, Theorem 4.6].

\section{Generalized S-Holomorphicity}

This section builds on the previous ones to obtain the main results of this article. In the first two paragraphs, we introduce a notion of s-holomorphicity valid for any weighted surface graph, generalizing the classical definition of Chelkak-Smirnov [6] which corresponds to the planar isoradial case. Moreover, we give three alternative viewpoints on this notion, each involving one of the three operators (Kac-Ward, Kasteleyn, Dirac) studied in Section 3 (Theorem 4.2 and Corollary 4.3). In the final two subsections, we show that several crucial properties of s-holomorphic functions on isoradial graphs extend to our setting. First, the minors of the Kac-Ward matrices are nothing but generalized spin-observables, which are automatically s-holomorphic (subsection 4.3). Also, it is possible to define a discrete version of the integral of the square of an s-holomorphic function (subsection 4.4).

4.1. The kernel of the Kac-Ward operator. As above, let $(\Gamma, x) \subset \Sigma$ be an arbitrary weighted surface graph. In this paragraph, we will analyse the kernel of the associated Kac-Ward operator $K W=K W(\Gamma, x)$, where the 1-cochain $\varphi$ is taken to be trivial. (The 
discussion below extends to any real-valued cochain, but the statements become unnecessarily cumbersome.)

First note that the corresponding Kasteleyn operator $K^{\omega}=K^{\omega}\left(C_{\Gamma}, y\right)$ restricts to a real operator $\mathrm{K}^{\omega}\left(C_{\Gamma}, y\right): \mathbb{R}^{B} \rightarrow \mathbb{R}^{W}$. The commutative diagram of Theorem 3.1 can therefore be completed into

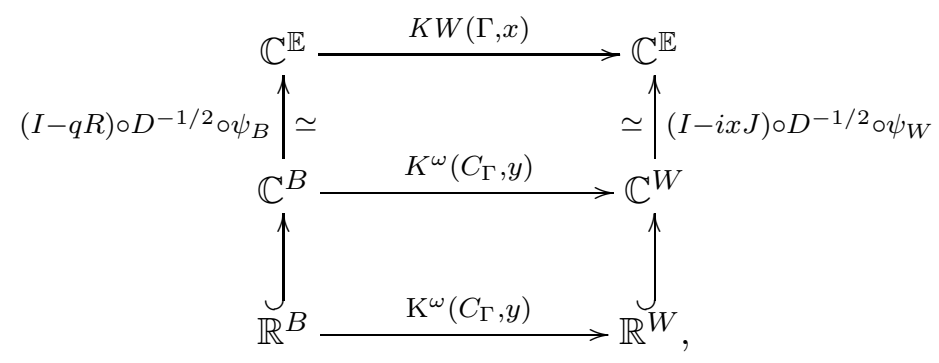

where the choice of the square root of $D$ is determined by the Kasteleyn orientation $\omega$.

For $e \in \mathbb{E}$, let us write

$$
\ell(e)=D_{e}^{-1 / 2} \cdot \mathbb{R}=\exp \left(-\frac{i}{2} a_{e}\right) \cdot \mathbb{R}
$$

which does not depend on the square root of $D$. By definition, both $\left(D^{-1 / 2} \circ \psi_{B}\right)\left(\mathbb{R}^{B}\right)$ and $\left(D^{-1 / 2} \circ \psi_{W}\right)\left(\mathbb{R}^{W}\right)$ coincide with the real vector subspace of $\mathbb{C}^{\mathbb{E}}$ given by

$$
\mathscr{L}=\left\{f \in \mathbb{C}^{\mathbb{E}} \mid f(e) \in \ell(e) \text { for all } e \in \mathbb{E}\right\} .
$$

Since $\ell(\bar{e})=i \ell(e), I-i x J$ leaves $\mathscr{L}$ invariant. Since $\ell(R(e))=q_{e}^{-1} \ell(e)$, the same holds for $I-q R$. Therefore, we obtain the following commutative diagram:

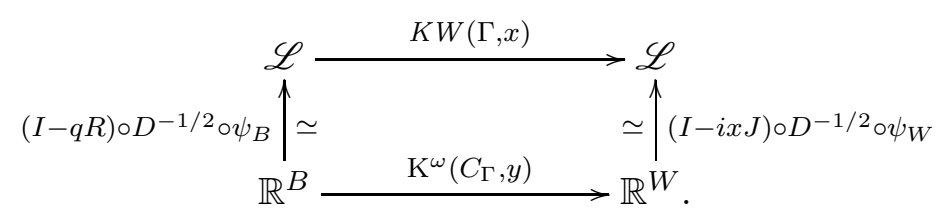

Given any real line $\ell \subset \mathbb{C}$, we shall denote by $\operatorname{Pr}(-; \ell): \mathbb{C} \rightarrow \ell$ the orthogonal projection onto $\ell$. We shall also simply write $\operatorname{Pr}(-; u)$ for the orthogonal projection onto the real line $u \cdot \mathbb{R}$ generated by $u \in \mathbb{C}^{*}$. Following Lis [34], let us consider the real linear map $S: \mathbb{C}^{\diamond} \rightarrow \mathscr{L}$ defined by

$$
(S F)(e)=\sin \left(\theta_{e} / 2\right) \operatorname{Pr}\left(F\left(z_{e}\right) ; \ell(e)\right)
$$

for $F \in \mathbb{C}^{\diamond}$ and $e \in \mathbb{E}$. The following result generalizes [34, Proposition 2.1], which deals with the planar isoradial case.

Proposition 4.1. Fix an element $F$ of $\mathbb{C}^{\diamond}$ and a vertex $v$ of $\Gamma$. Then, $f=S(F) \in \mathscr{L}$ satisfies $(K W f)(\bar{e})=0$ for all $e \in \mathbb{E}_{v}$ if and only if

$$
\operatorname{Pr}\left(F\left(z_{e}\right) ; i \exp \left(-\frac{i}{2}\left(a_{e}+\theta_{e}\right)\right)\right)=\operatorname{Pr}\left(F\left(z_{e^{\prime}}\right) ; i \exp \left(-\frac{i}{2}\left(a_{e^{\prime}}-\theta_{e^{\prime}}\right)\right)\right) \exp \left(\frac{i}{2}\left(\beta_{e}-\theta_{e}-\theta_{e^{\prime}}\right)\right)
$$

for all $e \in \mathbb{E}_{v}$, where $e^{\prime}$ stands for $R(e)$.

Proof. Using the notations of subsection 3.1, the first statement means that $(J \circ K W)(f)$ vanishes on $\mathbb{E}_{v}$. Consider the endomorphism of $\mathbb{C}^{\mathbb{E}}$ given by $(I-q R) x^{-1}$. It is clearly an isomorphism (recall the proof of Corollary 3.2), which splits as a direct sum of automorphisms 
of $\mathbb{C}^{\mathbb{E}_{v}}$ according to the partition $\mathbb{E}=\bigsqcup_{v} \mathbb{E}_{v}$. Therefore, $(J \circ K W)(f)$ vanishes on $\mathbb{E}_{v}$ if and only if $\left((I-q R) x^{-1} J K W\right)(f)$ vanishes on $\mathbb{E}_{v}$. In other words, for all $e \in \mathbb{E}_{v}$,

$$
\begin{aligned}
0= & \left((I-q R) x^{-1} J K W\right)(f)(e) \\
= & x_{e}^{-1}(K W f)(\bar{e})-q_{e} x_{e^{\prime}}^{-1}(K W f)\left(\overline{e^{\prime}}\right) \\
= & x_{e}^{-1}\left(f(\bar{e})-x_{e} \sum_{e^{\prime \prime} \in \mathbb{E}_{v} \backslash\{e\}} \exp \left(\frac{i}{2} \alpha_{\lambda}\left(\bar{e}, e^{\prime \prime}\right)\right) f\left(e^{\prime \prime}\right)\right) \\
& -\exp \left(\frac{i}{2} \beta_{e}\right) x_{e^{\prime}}^{-1}\left(f\left(\overline{e^{\prime}}\right)-x_{e^{\prime}} \sum_{e^{\prime \prime} \in \mathbb{E}_{v} \backslash\left\{e^{\prime}\right\}} \exp \left(\frac{i}{2} \alpha_{\lambda}\left(\overline{e^{\prime}}, e^{\prime \prime}\right)\right) f\left(e^{\prime \prime}\right)\right) \\
= & x_{e}^{-1} f(\bar{e})+i \exp \left(\frac{i}{2} \beta_{e}\right) f\left(e^{\prime}\right)-\exp \left(\frac{i}{2} \beta_{e}\right) x_{e^{\prime}}^{-1} f\left(\overline{e^{\prime}}\right)+i f(e) .
\end{aligned}
$$

Therefore, $f=S(F)$ satisfies $(K W f)(\bar{e})=0$ for all $e \in \mathbb{E}_{v}$ if and only if

$$
x_{e}^{-1} f(\bar{e})+i f(e)=\exp \left(\frac{i}{2} \beta_{e}\right)\left(x_{e^{\prime}}^{-1} f\left(\overline{e^{\prime}}\right)-i f\left(e^{\prime}\right)\right)
$$

for all $e \in \mathbb{E}_{v}$. Using the parametrization $x_{e}=\tan \left(\theta_{e} / 2\right)$ of the weights, the definition of $f=S(F)$, and the fact that $\ell(e)$ and $\ell(\bar{e})$ are orthogonal, the left-hand side reads

$$
\begin{aligned}
x_{e}^{-1} f(\bar{e})+i f(e) & =\sin \left(\theta_{e} / 2\right)^{-1}\left(\cos \left(\theta_{e} / 2\right) f(\bar{e})+i \sin \left(\theta_{e} / 2\right) f(e)\right) \\
& =\cos \left(\theta_{e} / 2\right) \operatorname{Pr}\left(F\left(z_{e}\right) ; \ell(\bar{e})\right)+i \sin \left(\theta_{e} / 2\right) \operatorname{Pr}\left(F\left(z_{e}\right) ; \ell(e)\right) \\
& =\operatorname{Pr}\left(\left(\cos \left(\theta_{e} / 2\right)+i \sin \left(\theta_{e} / 2\right)\right) F\left(z_{e}\right) ; \ell(\bar{e})\right) \\
& =\exp \left(\frac{i}{2} \theta_{e}\right) \operatorname{Pr}\left(F\left(z_{e}\right) ; \exp \left(-\frac{i}{2} \theta_{e}\right) \ell(\bar{e})\right) \\
& =\exp \left(\frac{i}{2} \theta_{e}\right) \operatorname{Pr}\left(F\left(z_{e}\right) ; i \exp \left(-\frac{i}{2}\left(a_{e}+\theta_{e}\right)\right)\right) .
\end{aligned}
$$

Similar considerations for the right-hand side lead to

$$
x_{e^{\prime}}^{-1} f\left(\overline{e^{\prime}}\right)-i f\left(e^{\prime}\right)=\exp \left(-\frac{i}{2} \theta_{e^{\prime}}\right) \operatorname{Pr}\left(F\left(z_{e^{\prime}}\right) ; i \exp \left(-\frac{i}{2}\left(a_{e^{\prime}}-\theta_{e^{\prime}}\right)\right)\right),
$$

and the proposition follows.

Note that the left-hand side of the equality in Proposition 4.1 can be written as

$$
\operatorname{Pr}\left(F\left(z_{e}\right) ; i \exp \left(-\frac{i}{2}\left(a_{e}+\theta_{e}\right)\right)\right)=-i \exp \left(-\frac{i}{2} \theta_{e}\right) D_{e}^{-1 / 2} \operatorname{Re}\left(i D_{e}^{1 / 2} \exp \left(\frac{i}{2} \theta_{e}\right) F\left(z_{e}\right)\right),
$$

while

$$
\operatorname{Pr}\left(F\left(z_{e^{\prime}}\right) ; i \exp \left(-\frac{i}{2}\left(a_{e^{\prime}}-\theta_{e^{\prime}}\right)\right)\right)=-i \exp \left(\frac{i}{2} \theta_{e^{\prime}}\right) D_{e^{\prime}}^{-1 / 2} R e\left(i D_{e^{\prime}}^{1 / 2} \exp \left(-\frac{i}{2} \theta_{e^{\prime}}\right) F\left(z_{e^{\prime}}\right)\right) .
$$

Therefore, this equality is equivalent to

$$
\operatorname{Re}\left(i D_{e}^{1 / 2} \exp \left(\frac{i}{2} \theta_{e}\right) F\left(z_{e}\right)\right)=\varepsilon(e) \operatorname{Re}\left(i D_{e^{\prime}}^{1 / 2} \exp \left(-\frac{i}{2} \theta_{e^{\prime}}\right) F\left(z_{e^{\prime}}\right)\right),
$$

where $\varepsilon(e)=q_{e} D_{e^{\prime}}^{-1 / 2} D_{e}^{1 / 2}= \pm 1$ depends on the Kasteleyn orientation $\omega$.

4.2. Three viewpoints on s-holomorphicity. Assume that $\Gamma$ is isoradially embedded in the plane with critical weights $x_{e}=\tan \left(\theta_{e} / 2\right)$. If $\lambda$ is chosen to be a constant vector field, then $\beta_{e}-\theta_{e}-\theta_{e^{\prime}}$ vanishes for all $e \in \mathbb{E}$. Therefore the equality in Proposition 4.1 simply reads

$$
\operatorname{Pr}\left(F\left(z_{e}\right) ; \ell_{e, e^{\prime}}\right)=\operatorname{Pr}\left(F\left(z_{e^{\prime}}\right) ; \ell_{e, e^{\prime}}\right),
$$

where $\ell_{e, e^{\prime}}=i \exp \left(-\frac{i}{2}\left(a_{e}+\theta_{e}\right)\right) \cdot \mathbb{R}=i \exp \left(-\frac{i}{2}\left(a_{e^{\prime}}-\theta_{e^{\prime}}\right)\right) \cdot \mathbb{R}$. This equality defines the fact that $\exp \left(i \frac{\pi}{4}\right) F$ is "s-holomorphic" [6]. Hence, the equality in Proposition 4.1, or equivalently, 
the fact of lying in the kernel of the Kac-Ward operator, should be understood as a generalized s-holomorphicity condition valid for any weighted surface graph. This motivates the following terminology.

Definition 6. Let $(\Gamma, x) \subset \Sigma$ be an arbitrary surface graph with weights parametrized by $x_{e}=\tan \left(\theta_{e} / 2\right)$. A function $F \in \mathbb{C}^{\diamond}$ is called s-holomorphic around $v \in V(\Gamma)$ if

$$
\operatorname{Pr}\left(F\left(z_{e}\right) ;\left[i \exp \left(i\left(a_{e}+\theta_{e}\right)\right)\right]^{-\frac{1}{2}}\right)=\operatorname{Pr}\left(F\left(z_{e^{\prime}}\right) ;\left[i \exp \left(i\left(a_{e^{\prime}}-\theta_{e^{\prime}}\right)\right)\right]^{-\frac{1}{2}}\right) \exp \left(\frac{i}{2}\left(\beta_{e}-\theta_{e}-\theta_{e^{\prime}}\right)\right)
$$

for all $e \in \mathbb{E}_{v}$, where $e^{\prime}$ stands for $R(e)$.

Note that the choice of the square root is irrelevant.

We shall now build on the results of Section 3 to give three viewpoints on the generalized notion of s-holomorphicity defined above. Recall that a Kasteleyn orientation $\omega$ on $C_{\Gamma} \subset \Sigma$ determines a square root $D^{1 / 2}=\left(D_{e}^{1 / 2}\right)_{e \in \mathbb{E}}$ of $D=\left(D_{e}\right)_{e \in \mathbb{E}}=\left(\exp \left(i a_{e}\right)\right)_{e \in \mathbb{E}}$ as explained in Theorem [3.1, and that these square roots satisfy $D_{R(e)}^{1 / 2}=\varepsilon(e) q_{e} D_{e}^{1 / 2}$ for all $e \in \mathbb{E}$, with $\varepsilon(e)= \pm 1$. This allows us to define $T: \mathbb{C}^{\diamond} \rightarrow \mathbb{R}^{B}$ by

$$
(T F)(b)=\sum_{e \in \mathbb{E}_{v}} \operatorname{Re}\left(\varepsilon_{b}(e) D_{e}^{1 / 2} \sin \left(\theta_{e} / 2\right) F\left(z_{e}\right)\right),
$$

where $v \in V(\Gamma)$ denotes the vertex of $\Gamma$ closest to $b \in B$, i.e. $v=o\left(e_{0}\right)$ if $b=\psi_{B}\left(e_{0}\right)$, and $\varepsilon_{b}(e)=\varepsilon\left(e_{0}\right) \varepsilon\left(R\left(e_{0}\right)\right) \cdots \varepsilon\left(R^{k-1}\left(e_{0}\right)\right)$ if $e=R^{k}\left(e_{0}\right), 0 \leq k<\operatorname{deg}(v)$. Finally, set $T^{\prime}:=$ $\exp \left(\frac{i}{2} \theta_{B}\right) \circ T$, where $\theta_{B}=\left(\theta_{\psi_{B}^{-1}(b)}\right)_{b \in B}$.

Theorem 4.2. The maps $S: \mathbb{C}^{\diamond} \rightarrow \mathscr{L}, T: \mathbb{C}^{\diamond} \rightarrow \mathbb{R}^{B}$ and $T^{\prime}: \mathbb{C}^{\diamond} \rightarrow T^{\prime}\left(\mathbb{C}^{\diamond}\right) \subset \mathbb{C}^{B}$ are $\mathbb{R}$-linear isomorphisms such that, for any $F \in \mathbb{C}^{\diamond}$, the following are equivalent:

(i) $\exp \left(i \frac{\pi}{4}\right) F$ is s-holomorphic.

(ii) $S(F) \in \mathscr{L}$ lies in the kernel of the Kac-Ward operator $K W(\Gamma, x)$.

(iii) $T(F) \in \mathbb{R}^{B}$ lies in the kernel of the Kasteleyn operator $\mathrm{K}^{\omega}\left(C_{\Gamma}, y\right)$.

Furthermore, if $\Gamma$ is isoradially embedded in a flat surface $\Sigma$ with critical weights, then these three conditions are equivalent to:

(iv) $T^{\prime}(F) \in \mathbb{C}^{B}$ lies in the kernel of the discrete $\bar{\partial}$-operator $\bar{\partial}_{C}^{\varphi_{\omega}}$.

Proof. The map $S: \mathbb{C}^{\diamond} \rightarrow \mathscr{L}$ is clearly an isomorphism of real vector spaces, with inverse $\left(S^{-1} f\right)(z)=\sin \left(\theta_{e} / 2\right)^{-1}(f(e)+f(\bar{e}))$ for $f \in \mathbb{C}^{\mathbb{E}}$ and $z=z_{e} \in \diamond$. By definition, $\exp \left(i \frac{\pi}{4}\right) F$ is s-holomorphic if and only if $F$ satisfies the equality in Proposition 4.1. hence, $(i)$ and $(i i)$ are equivalent by this proposition.

Now, let us define $T_{0}: \mathbb{C}^{\diamond} \rightarrow \mathbb{R}^{B}$ as the unique $\mathbb{R}$-linear isomorphism such that $(I-q R) \circ$ $D^{-1 / 2} \circ \psi_{B} \circ T_{0}=S$. By Theorem 3.1 and the discussion in subsection 4.1, we have the commutative diagram

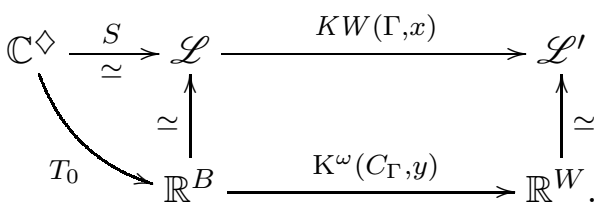


This implies that $K W(S F)=0$ if and only if $T_{0}(F)$ lies in the kernel of $\mathrm{K}^{\omega}\left(C_{\Gamma}, y\right)$. Therefore, it remains to check that $T_{0}$ coincides with the map $T$ defined before the statement of the theorem (up to a real scalar factor). To do so, first observe that the automorphism $I-q R$ of $\mathbb{C}^{\mathbb{E}}$ splits as a direct sum $\bigoplus_{v \in V(\Gamma)}(I-q R)_{v}$ according to the partition $\mathbb{E}=\bigsqcup_{v \in V(\Gamma)} \mathbb{E}_{v}$, and that $2(I-q R)_{v}^{-1}=I+q R+\cdots+(q R)^{\operatorname{deg}(v)-1}$. This leads to the following computation, where $F \in \mathbb{C}^{\diamond}, b=\psi_{B}(e) \in B$ and $v=o(e) \in V(\Gamma)$ :

$$
\begin{aligned}
2\left(T_{0} F\right)(b) & =2\left(\psi_{B}^{-1} \circ D^{1 / 2} \circ(I-q R)^{-1} \circ S\right)(F)(b) \\
& =2 D_{e}^{1 / 2}(I-q R)_{v}^{-1}(S F)(e) \\
& =D_{e}^{1 / 2} \sum_{k=0}^{\operatorname{deg}(v)-1} q_{e} \cdots q_{R^{k-1}(e)}(S F)\left(R^{k}(e)\right) \\
& =\sum_{e^{\prime} \in \mathbb{E}} \varepsilon_{b}\left(e^{\prime}\right) D_{e^{\prime}}^{1 / 2}(S F)\left(e^{\prime}\right) \\
& =\sum_{e^{\prime} \in \mathbb{E}} \varepsilon_{b}\left(e^{\prime}\right) D_{e^{\prime}}^{1 / 2} \sin \left(\theta_{e^{\prime}} / 2\right) \operatorname{Pr}\left(F\left(z_{e^{\prime}}\right) ; \ell\left(e^{\prime}\right)\right) \\
& =\sum_{e^{\prime} \in \mathbb{E}_{v}} \operatorname{Re}\left(\varepsilon_{b}\left(e^{\prime}\right) D_{e^{\prime}}^{1 / 2} \sin \left(\theta_{e^{\prime}} / 2\right) F\left(z_{e^{\prime}}\right)\right) .
\end{aligned}
$$

This shows that the map $T=2 T_{0}$ is an isomorphism, and that conditions (ii) and (iii) are equivalent. The map $T^{\prime}=\exp \left(\frac{i}{2} \theta_{B}\right) \circ T$ being the composition of two isomorphisms, it is one as well.

Finally, let us assume that $\Gamma$ is isoradially embedded in a flat surface $\Sigma$ with weights $x_{e}=\tan \left(\theta_{e} / 2\right)$. By Proposition [3.6, we have the commutative diagram

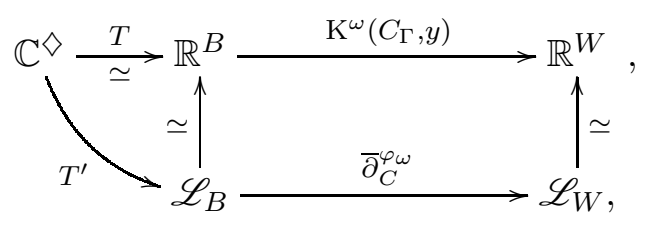

where $\mathscr{L}_{B}=\exp \left(\frac{i}{2} \theta_{B}\right)\left(\mathbb{R}^{B}\right)$ and $\mathscr{L}_{W}=\exp \left(\frac{i}{2} \theta_{W}\right)\left(\mathbb{R}^{W}\right)$. Therefore, $T(F)$ lies in the kernel of $\mathrm{K}^{\omega}\left(C_{\Gamma}, y\right)$ if and only if $T^{\prime}(F)$ lies in the kernel of $\bar{\partial}_{C}^{\varphi_{\omega}}$.

This theorem admits the following corollary.

Corollary 4.3. Let $s \mathscr{O}$ denote the real vector subspace of $\mathbb{C}^{\diamond}$ consisting of functions $F$ such that $\exp \left(i \frac{\pi}{4}\right) F$ is s-holomorphic.

(i) The map $S: \mathbb{C}^{\diamond} \rightarrow \mathbb{C}^{\mathbb{E}}$ restricts to an $\mathbb{R}$-linear isomorphism $s \mathscr{O} \simeq \mathscr{L} \cap \operatorname{ker}(K W)$.

(ii) The map $\widetilde{T}: \mathbb{C}^{\diamond} \rightarrow \mathbb{R}^{B}$ defined by

$$
(\widetilde{T} F)(b)=\operatorname{Re}\left(i D_{e}^{1 / 2} \exp \left(-\frac{i}{2} \theta_{e}\right) F\left(z_{e}\right)\right)
$$

for $F \in \mathbb{C}^{\diamond}$ and $b=\psi_{B}(e) \in B$ restricts to an isomorphism $s \mathscr{O} \simeq \operatorname{ker}\left(\mathrm{K}^{\omega}\right)$.

(iii) If $\Gamma$ is isoradially embedded in a flat surface $\Sigma$ with critical weights, then the map $\widetilde{T}^{\prime}: \mathbb{C}^{\diamond} \rightarrow \mathbb{C}^{B}$ defined by

$$
\left(\widetilde{T}^{\prime} F\right)(b)=i D_{e}^{1 / 2} \operatorname{Pr}\left(F\left(z_{e}\right) ; i \exp \left(-\frac{i}{2}\left(a_{e}-\theta_{e}\right)\right)\right)
$$


for $F \in \mathbb{C}^{\diamond}$ and $b=\psi_{B}(e) \in B$ restricts to an isomorphism $s \mathscr{O} \simeq \operatorname{ker}\left(\bar{\partial}_{C}^{\varphi_{\omega}}\right) \cap \widetilde{T}^{\prime}\left(\mathbb{C}^{\diamond}\right)$.

Note that in the planar isoradial case, $\widetilde{T}(F)$ is the so-called real spinor associated to $F$ by Chelkak-Smirnov in [6, Lemma 3.4]. To be more precise, the vertices of $C_{\Gamma}$ play the role of the vertices of the double cover $\widehat{\Upsilon}$ in [6], the choice of this double cover, or spin structure [36, Definition 9], corresponding to the choice of a Kasteleyn orientation on $C_{\Gamma}$ (see [11]). Furthermore, the fact that $\widetilde{T}(F)$ lies in the kernel of the Kasteleyn operator translates into the propagation equation [6, Equation (3.6)], or the Dirac equation [36, Equation (4.5)] (see also [15, Section 4.2]). Therefore, the statement (ii) above should be understood as a generalized propagation equation, valid for any weighted surface graph.

Proof of Corollary 4.3. The first point follows immediately from Theorem 4.2, For the second point, it remains to check that the restriction of $T$ to $s \mathscr{O}$ coincides with the map $\widetilde{T}$ (up to a real scalar factor). This follows from the computation below, where we make use of Equation $(\star)$ in the fourth equality, and of the notation $e^{\prime}=R(e)$ :

$$
\begin{aligned}
\left((I-q R) \circ D^{-1 / 2} \circ \psi_{B} \circ \widetilde{T}\right)(F)(e)=\left(D^{-1 / 2} \circ \psi_{B} \circ \widetilde{T}\right)(F)(e)-q_{e}\left(D^{-1 / 2} \circ \psi_{B} \circ \widetilde{T}\right)(F)\left(e^{\prime}\right) \\
=D_{e}^{-1 / 2}(\widetilde{T} F)\left(\psi_{B}(e)\right)-q_{e} D_{e^{\prime}}^{-1 / 2}(\widetilde{T} F)\left(\psi_{B}\left(e^{\prime}\right)\right) \\
=D_{e}^{-1 / 2} \operatorname{Re}\left(i D_{e}^{1 / 2} \exp \left(-\frac{i}{2} \theta_{e}\right) F\left(z_{e}\right)\right)-q_{e} D_{e^{\prime}}^{-1 / 2} \operatorname{Re}\left(i D_{e^{\prime}}^{1 / 2} \exp \left(-\frac{i}{2} \theta_{e^{\prime}}\right) F\left(z_{e^{\prime}}\right)\right) \\
=D_{e}^{-1 / 2} \operatorname{Re}\left(i D_{e}^{1 / 2} \exp \left(-\frac{i}{2} \theta_{e}\right) F\left(z_{e}\right)\right)-D_{e}^{-1 / 2} \operatorname{Re}\left(i D_{e}^{1 / 2} \exp \left(\frac{i}{2} \theta_{e}\right) F\left(z_{e}\right)\right) \\
=D_{e}^{-1 / 2} \operatorname{Re}\left(D_{e}^{1 / 2} 2 \sin \left(\theta_{e} / 2\right) F\left(z_{e}\right)\right) \\
=2 \sin \left(\theta_{e} / 2\right) \operatorname{Pr}\left(F\left(z_{e}\right) ; D_{e}^{-1 / 2} \cdot \mathbb{R}\right) \\
=2(S F)(e) .
\end{aligned}
$$

As for the third point, it follows from the second one and from the computation below:

$$
\begin{aligned}
\left(\widetilde{T}^{\prime} F\right)(b) & =\left(\exp \left(\frac{i}{2} \theta_{B}\right) \circ \widetilde{T}\right)(F)(b) \\
& =\exp \left(\frac{i}{2} \theta_{e}\right) \operatorname{Re}\left(i D_{e}^{1 / 2} \exp \left(-\frac{i}{2} \theta_{e}\right) F\left(z_{e}\right)\right) \\
& =i D_{e}^{1 / 2} \operatorname{Pr}\left(F\left(z_{e}\right) ; i \exp \left(-\frac{i}{2}\left(a_{e}-\theta_{e}\right)\right)\right) .
\end{aligned}
$$

This concludes the proof.

Let us conclude this paragraph on s-holomorphicity with one final result. It is natural to wonder about the connection between (generalized) s-holomorphicity and continuous holomorphicity, especially on Riemann surfaces. Obviously, there is no such relation in full generality when the weights of the graph are not related to the conformal structure of the underlying surface. In the critical isoradial case however, it is possible to use Corollary 4.3 above together with Theorems 2.5 and 3.5 of [9] to obtain convergence results. The precise definition of all the objects involved being quite cumbersome, we refer the reader to [9] for details.

Corollary 4.4. Let $\Sigma$ be a flat surface with cone-type singularities and trivial holonomy. Consider a sequence $\Gamma_{n}$ of graphs $\delta_{n}$-isoradially embedded in $\Sigma$ with $\lim _{n} \delta_{n}=0$, such that all rhombi angles of all these isoradial graphs belong to the interval $[\eta, \pi-\eta]$ for some $\eta>0$. 
Finally, let $F_{n} \in \mathbb{C}^{\diamond_{n}}$ be a sequence of functions such that $\exp \left(i \frac{\pi}{4}\right) F_{n}$ is s-holomorphic (with respect to a constant vector field).

(i) Assume that $F_{n} \in \mathbb{C}^{\diamond_{n}}$ converges to $F: \Sigma \rightarrow \mathbb{C}$ in the following sense: for any sequence $z_{n} \in \diamond_{n}$ converging in $\Sigma, F_{n}\left(z_{n}\right)$ converges to $F\left(\lim _{n} z_{n}\right)$ in $\mathbb{C}$. Then $F$ is holomorphic in $\Sigma$.

(ii) Assume that all cone angles are odd multiples of $2 \pi$ and that the discrete spinors $\widetilde{T}^{\prime} F_{n} \in \mathbb{C}^{B_{n}}$ converge to a section $\psi$ of a fixed spin structure, interpreted as a line bundle $L \rightarrow \Sigma$. Then, $\psi$ is a holomorphic spinor.

Proof. Since $\Sigma$ has trivial holonomy, it makes sense to talk about a constant vector field on $\Sigma$. With respect to such a vector field, the definition of s-holomorphicity for isoradial graphs is the usual one. As in [6, Lemma 3.2], one then checks that if $F \in \mathbb{C}^{\diamond}$ is s-holomorphic, then $F$ lies in the kernel of the discrete $\bar{\partial}$-operator $\bar{\partial}_{D}: \mathbb{C}^{B} \rightarrow \mathbb{C}^{W}$ associated with the double graph $D_{\Gamma}$ with bipartite structure $B=\diamond$ and $W=V(\Gamma) \cup V\left(\Gamma^{*}\right)$. The first statement now follows from [9, Theorem 2.5] applied to the bipartite graphs $D_{\Gamma_{n}}$. By Corollary 4.3 above, $\widetilde{T}^{\prime} F_{n}$ lies in the kernel of the discrete $\bar{\partial}$-operator $\bar{\partial}_{C_{n}}^{\varphi_{n}}: \mathbb{C}^{B_{n}} \rightarrow \mathbb{C}^{W_{n}}$ associated with the graph $C_{\Gamma_{n}}$, where $\varphi_{n}$ is a discrete spin structure on $C_{\Gamma_{n}}$ which can be chosen to represent the fixed spin structure $L$. The second statement now follows from [9, Theorem 3.12] applied to the bipartite graphs $C_{\Gamma_{n}}$. (Note that even though some rhombi of $C_{\Gamma_{n}}$ are degenerate, Lemma 2.7 of [9] still holds in this setting, and the theorem does apply.)

4.3. The inverse Kac-Ward operator. Let $(\Gamma, x) \subset \Sigma$ be an arbitrary surface graph, and as before, let us consider a fixed vector field $\lambda$ on $\Sigma$ with zeroes of even index in $\Sigma \backslash \Gamma$. Recall that the associated Kac-Ward operator $K W=K W^{\lambda}: \mathbb{C}^{\mathbb{E}} \rightarrow \mathbb{C}^{\mathbb{E}}$ can be defined by $\left(K W^{\lambda} f\right)(e)=\sum_{e^{\prime} \in \mathbb{E}} K W^{\lambda}\left(e, e^{\prime}\right) f\left(e^{\prime}\right)$ for $f \in \mathbb{C}^{\mathbb{E}}$ and $e \in \mathbb{E}$, with coefficients

$$
K W^{\lambda}\left(e, e^{\prime}\right)= \begin{cases}1 & \text { if } e=e^{\prime} \\ -x_{e} \exp \left(\frac{i}{2} \alpha_{\lambda}\left(e, e^{\prime}\right)\right) & \text { if } o\left(e^{\prime}\right)=t(e) \text { but } e^{\prime} \neq \bar{e} \\ 0 & \text { else, }\end{cases}
$$

with $\alpha_{\lambda}\left(e, e^{\prime}\right)$ the rotation angle in radians of the velocity vector field along $e$ followed by $e^{\prime}$ with respect to the vector field $\lambda$.

As mentioned in subsection 2.4, its determinant is the square of a polynomial in the weight variables. The precise result is most conveniently stated using the terminology of homology, that we now very briefly recall. Given a surface graph $\Gamma \subset \Sigma$, let $C_{0}$ (resp. $C_{1}, C_{2}$ ) denote the $\mathbb{Z}_{2}$-vector space with basis the set of vertices (resp. edges, faces) of $\Gamma \subset \Sigma$. Elements of $C_{i}$ are called $i$-chains. Also, let $\partial_{2}: C_{2} \rightarrow C_{1}$ and $\partial_{1}: C_{1} \rightarrow C_{0}$ denote the boundary operators defined in the obvious way. Since $\partial_{1} \circ \partial_{2}$ vanishes, the space of 1-cycles $\operatorname{ker}\left(\partial_{1}\right)$ contains the space $\partial_{2}\left(C_{2}\right)$ of 1-boundaries. The first homology space $H_{1}\left(\Sigma ; \mathbb{Z}_{2}\right):=\operatorname{ker}\left(\partial_{1}\right) / \partial_{2}\left(C_{2}\right)$ turns out not to depend on $\Gamma$, but only on $\Sigma$ : it has dimension $2 g$, where $g$ is the genus of the closed connected orientable surface $\Sigma$. Note that the intersection of curves defines a non-degenerate bilinear form on $H_{1}\left(\Sigma ; \mathbb{Z}_{2}\right)$, that will be denoted by $(\alpha, \beta) \mapsto \alpha \cdot \beta$.

We shall also need the following classical result of Johnson [22]: given a vector field $\lambda$ on $\Sigma$ with zeroes of even index and a piecewise smooth curve $\gamma$ in $\Sigma$ avoiding the zeroes of $\lambda$, let $\operatorname{rot}_{\lambda}(\gamma) \in 2 \pi \mathbb{Z}$ denote the rotation angle of the velocity vector field of $\gamma$ with respect to 
$\lambda$. Then, given a homology class $\alpha \in H_{1}\left(\Sigma ; \mathbb{Z}_{2}\right)$ represented by the disjoint union of oriented simple closed curves $\gamma_{j}$, the equality

$$
(-1)^{q_{\lambda}(\alpha)}=\prod_{j}-\exp \left(\frac{i}{2} \operatorname{rot}_{\lambda}\left(\gamma_{j}\right)\right)
$$

gives a well-defined quadratic form on $H_{1}\left(\Sigma ; \mathbb{Z}_{2}\right)$, i.e. a map $q_{\lambda}: H_{1}\left(\Sigma ; \mathbb{Z}_{2}\right) \rightarrow \mathbb{Z}_{2}$ such that $q_{\lambda}(\alpha+\beta)=q_{\lambda}(\alpha)+q_{\lambda}(\beta)+\alpha \cdot \beta$ for all $\alpha, \beta \in H_{1}\left(\Sigma ; \mathbb{Z}_{2}\right)$. This implies in particular that, for any oriented closed curve $\gamma$ with $t(\gamma)$ transverse self-intersection points,

$$
-\exp \left(\frac{i}{2} \operatorname{rot}_{\lambda}(\gamma)\right)=(-1)^{q_{\lambda}(\gamma)+t(\gamma)} .
$$

(This can be checked by smoothing out the intersection points according to the orientation of $\gamma$ and using the definition of $q_{\lambda}$.)

Coming back to the Kac-Ward determinant, it was showed in [7] that

$$
\operatorname{det}\left(K W^{\lambda}\right)=\left(\sum_{\xi \in \mathscr{E}}(-1)^{q_{\lambda}(\xi)} x(\xi)\right)^{2}
$$

where $\mathscr{E}$ denotes the set of 1-cycles in $\Gamma$, that is, the set of subgraphs $\xi$ of $\Gamma$ such that each vertex of $\Gamma$ is adjacent to an even number of edges of $\xi$, and $x(\xi)$ stands for $\prod_{e \in \xi} x_{e}$. Since $q_{\lambda}$ is well-defined in homology, this equality can be rewritten

$$
\operatorname{det}\left(K W^{\lambda}\right)^{1 / 2}=\sum_{\alpha \in H_{1}\left(\Sigma ; \mathbb{Z}_{2}\right)}(-1)^{q_{\lambda}(\alpha)} \sum_{\substack{\xi \in \mathscr{E} \\[\xi]=\alpha}} x(\xi)
$$

which easily leads to the generalized Kac-Ward formula (1) for $Z^{I s i n g}=\sum_{\xi \in \mathscr{E}} x(\xi)$.

Let us introduce some more notation. Let $\widetilde{\Gamma}$ be the graph obtained from $\Gamma$ by adding the vertex $z_{e}$ in the middle of each edge $e \in E$. Given $e, e^{\prime} \in \mathbb{E}$, we shall denote by $\mathscr{E}\left(e, e^{\prime}\right)$ the set of subgraphs $\xi \subset \widetilde{\Gamma}$ that contain the half-edges $\left(z_{e}, t(e)\right)$ and $\left(o\left(e^{\prime}\right), z_{e^{\prime}}\right)$, and such that each vertex in $V(\widetilde{\Gamma}) \backslash\left\{z_{e}, z_{e^{\prime}}\right\}$ is adjacent to an even number of edges of $\xi$. Given any $\xi \in \mathscr{E}\left(e, e^{\prime}\right)$, one can resolve its crossings to obtain a disjoint union $\gamma_{\xi}^{0} \sqcup \gamma_{\xi}$, where $\gamma_{\xi}^{0}$ consists in a family of disjoint simple closed curves and $\gamma_{\xi}$ is an oriented simple curve from $z_{e}$ to $z_{e^{\prime}}$. Note that the complex number $(-1)^{q_{\lambda}\left(\gamma_{\xi}^{0}\right)} \exp \left(\frac{i}{2} \operatorname{rot}_{\lambda}\left(\gamma_{\xi}\right)\right)$ is unchanged by merging curves in the family above; therefore, it does not depend on the choice of the smoothing of $\xi$.

Definition 7. Let $F^{\lambda}: \mathbb{C}^{\mathbb{E}} \rightarrow \mathbb{C}^{\mathbb{E}}$ be defined by $\left(F^{\lambda} g\right)(e)=\sum_{e^{\prime} \in \mathbb{E}} F^{\lambda}\left(e, e^{\prime}\right) g\left(e^{\prime}\right)$ for $g \in \mathbb{C}^{\mathbb{E}}$ and $e \in \mathbb{E}$, with coefficients

$$
F^{\lambda}\left(e, e^{\prime}\right)= \begin{cases}\sum_{e \notin \xi \in \mathscr{E}}(-1)^{q_{\lambda}(\xi)} x(\xi) & \text { if } e=e^{\prime} \\ \sum_{\xi \in \mathscr{E}\left(e, e^{\prime}\right)}(-1)^{q_{\lambda}\left(\gamma_{\xi}^{0}\right)} \exp \left(\frac{i}{2} \operatorname{rot}_{\lambda}\left(\gamma_{\xi}\right)\right) x_{e} x(\xi) & \text { else, }\end{cases}
$$

where $x(\xi)$ stands for the product of the weights of all the edges of $\Gamma$ contained in $\xi$.

We are now ready to state the main result of this paragraph. Note that in the planar case, it was obtained independently (and announced first) by Lis [34].

Theorem 4.5. For any $\lambda, K W^{\lambda} \circ F^{\lambda}=\operatorname{det}\left(K W^{\lambda}\right)^{1 / 2} \cdot I d_{\mathbb{C}^{\mathbb{E}}}$.

The following elementary lemma will be useful. 
Lemma 4.6. Let $\xi$ be a graph containing an edge $e_{1}$ adjacent to a vertex $v$ of even degree. For any edge $e \neq e_{1}$ adjacent to $v$, let $n_{\xi}\left(e_{1}, e\right)$ denote the number of edges of $\xi$ adjacent to $v$ strictly between $e_{1}$ and $e$ (on one given side). Then, $\sum_{v \in e \neq e_{1}}(-1)^{n_{\xi}\left(e_{1}, e\right)}=1$.

Proof. First note that the degree of $v$ being even, the parity of $n_{\xi}\left(e_{1}, e\right)$ does not depend on the choice of the side. Enumerating the edges $e_{1}, e_{2}, \ldots, e_{2 d}$ cyclically around $v$, we obtain $\sum_{v \in e \neq e_{1}}(-1)^{n_{\xi}\left(e_{1}, e\right)}=\sum_{j=2}^{2 d}(-1)^{j-2}=1$.

Proof of Theorem 4.5. Let $\lambda$ be a fixed vector field, that will be dropped from the notations for simplicity. We need to check that the coefficients of $K W$ and of $F$ satisfy the relation

$$
\sum_{e \in \mathbb{E}} K W\left(e_{1}, e\right) F\left(e, e_{2}\right)=\delta_{e_{1}, e_{2}} \sum_{\xi \in \mathscr{E}}(-1)^{q(\xi)} x(\xi)
$$

for all $e_{1}, e_{2} \in \mathbb{E}$. We will distinguish three cases.

Let us first assume that $e_{1}$ and $e_{2}$ coincide. In this case, $\sum_{e \in \mathbb{E}} K W\left(e_{1}, e\right) F\left(e, e_{1}\right)$ is by definition equal to

$$
\sum_{\substack{\xi^{\prime} \in \mathscr{E} \\ e_{1} \notin \xi^{\prime}}}(-1)^{q\left(\xi^{\prime}\right)} x\left(\xi^{\prime}\right)-\sum_{\substack{o(e)=t\left(e_{1}\right) \\ e \neq \bar{e}_{1}}} \sum_{\xi \in \mathscr{E}\left(e, e_{1}\right)}(-1)^{q\left(\gamma_{\xi}^{0}\right)} \exp \left(\frac{i}{2}\left(\alpha\left(e_{1}, e\right)+\operatorname{rot}\left(\gamma_{\xi}\right)\right) x_{e_{1}} x_{e} x(\xi) .\right.
$$

Given $\xi \in \mathscr{E}\left(e, e_{1}\right)$, let $\xi^{\prime}$ (resp. $\gamma_{\xi}^{\prime}$ ) denote the 1-cycle obtained from $\xi$ (resp. $\gamma_{\xi}$ ) by adding the half-edges $\left(z_{e_{1}}, t\left(e_{1}\right)\right)$ and $\left(o(e), z_{e}\right)$. The assignment $\xi \mapsto \xi^{\prime}$ defines a map from $\mathscr{E}\left(e, e_{1}\right)$ to the set of 1-cycles containing $e_{1}$, and it satisfies $x_{e_{1}} x_{e} x(\xi)=x\left(\xi^{\prime}\right)$. Furthermore,

$$
-\exp \left(\frac{i}{2}\left(\alpha\left(e_{1}, e\right)+\operatorname{rot}\left(\gamma_{\xi}\right)\right)\right)=-\exp \left(\frac{i}{2} \operatorname{rot}\left(\gamma_{\xi}^{\prime}\right)\right)=(-1)^{q\left(\gamma_{\xi}^{\prime}\right)+t\left(\gamma_{\xi}^{\prime}\right)} .
$$

The homology class of $\gamma_{\xi}^{\prime}$ satisfies $\left[\gamma_{\xi}^{\prime}\right]=\left[\gamma_{\xi}^{0}\right]+\left[\xi^{\prime}\right]$. Since $q$ is a quadratic form, we get the following equalities modulo 2 :

$$
q\left(\gamma_{\xi}^{0}\right)+q\left(\gamma_{\xi}^{\prime}\right)+t\left(\gamma_{\xi}^{\prime}\right)=q\left(\xi^{\prime}\right)+\gamma_{\xi}^{0} \cdot \xi^{\prime}+t\left(\gamma_{\xi}^{\prime}\right)=q\left(\xi^{\prime}\right)+\gamma_{\xi}^{0} \cdot \gamma_{\xi}^{\prime}+t\left(\gamma_{\xi}^{\prime}\right)=q\left(\xi^{\prime}\right)+n_{\xi^{\prime}}\left(e_{1}, e\right),
$$

where $n_{\xi^{\prime}}\left(e_{1}, e\right)$ denotes the number of edges of $\xi^{\prime}$ adjacent to $t\left(e_{1}\right)=o(e)=: v$ strictly between $e_{1}$ and $e$. Using Lemma 4.6, we get

$$
\begin{aligned}
\sum_{e \in \mathbb{E}} K W\left(e_{1}, e\right) F\left(e, e_{1}\right) & =\sum_{\substack{\xi^{\prime} \in \mathscr{E} \\
e_{1} \notin \xi^{\prime}}}(-1)^{q\left(\xi^{\prime}\right)} x\left(\xi^{\prime}\right)+\sum_{\substack{\xi^{\prime} \in \mathscr{E} \\
e_{1} \in \xi^{\prime}}}(-1)^{q\left(\xi^{\prime}\right)} x\left(\xi^{\prime}\right)\left(\sum_{\substack{e \in \xi^{\prime} \\
v \in e \neq e_{1}}}(-1)^{n_{\xi^{\prime}}\left(e_{1}, e\right)}\right) \\
& =\sum_{\xi^{\prime} \in \mathscr{E}}(-1)^{q\left(\xi^{\prime}\right)} x\left(\xi^{\prime}\right)
\end{aligned}
$$

as expected.

Let us now consider the case of $e_{1} \neq e_{2}$ with $t\left(e_{1}\right) \neq o\left(e_{2}\right)$. By definition,

$$
\begin{aligned}
\sum_{e \in \mathbb{E}} K W\left(e_{1}, e\right) F\left(e, e_{2}\right)= & \sum_{\xi^{\prime} \in \mathscr{E}\left(e_{1}, e_{2}\right)}(-1)^{q\left(\gamma_{\xi^{\prime}}^{0}\right)} \exp \left(\frac{i}{2} \operatorname{rot}\left(\gamma_{\xi^{\prime}}\right)\right) x\left(\xi^{\prime}\right)+ \\
& -\sum_{\substack{o(e)=t\left(e_{1}\right) \\
e \neq \bar{e}_{1}}} \sum_{\xi \in \mathscr{E}\left(e, e_{2}\right)}(-1)^{q\left(\gamma_{\xi}^{0}\right)} \exp \left(\frac{i}{2}\left(\alpha\left(e_{1}, e\right)+\operatorname{rot}\left(\gamma_{\xi}\right)\right)\right) x_{e_{1}} x_{e} x(\xi) .
\end{aligned}
$$


Given $\xi \in \mathscr{E}\left(e, e_{2}\right)$, let $\xi^{\prime}$ (resp. $\gamma_{\xi}^{\prime}$ ) denote the subgraph (resp. the oriented curve) obtained from $\xi$ (resp. $\gamma_{\xi}$ ) by adding the half-edges $\left(z_{e_{1}}, t\left(e_{1}\right)\right)$ and $\left(o(e), z_{e}\right)$. This defines a map from $\mathscr{E}\left(e, e_{2}\right)$ to $\mathscr{E}\left(e_{1}, e_{2}\right)$ such that $x_{e_{1}} x_{e} x(\xi)=x\left(\xi^{\prime}\right)$. Furthermore,

$$
-\exp \left(\frac{i}{2}\left(\alpha\left(e_{1}, e\right)+\operatorname{rot}\left(\gamma_{\xi}\right)-\operatorname{rot}\left(\gamma_{\xi^{\prime}}\right)\right)=-\exp \left(\frac{i}{2} \operatorname{rot}(\gamma)\right)=(-1)^{q(\gamma)+t(\gamma)},\right.
$$

where $\gamma$ is the closed oriented curve obtained by following $\gamma_{\xi}^{\prime}$ and then $-\gamma_{\xi^{\prime}}$. Since its homology class satisfies $[\gamma]=\left[\gamma_{\xi}^{\prime}+\gamma_{\xi^{\prime}}\right]=\left[\gamma_{\xi}^{0}\right]+\left[\gamma_{\xi^{\prime}}^{0}\right]$, we have

$$
q\left(\gamma_{\xi}^{0}\right)+q(\gamma)+t(\gamma)=q\left(\gamma_{\xi^{\prime}}^{0}\right)+\gamma_{\xi}^{0} \cdot \gamma_{\xi^{\prime}}^{0}+t(\gamma)=q\left(\gamma_{\xi^{\prime}}^{0}\right)+n_{\xi^{\prime}}\left(e_{1}, e\right) .
$$

This leads to

$$
\sum_{e \in \mathbb{E}} K W\left(e_{1}, e\right) F\left(e, e_{2}\right)=\sum_{\xi^{\prime} \in \mathscr{E}\left(e_{1}, e_{2}\right)}(-1)^{q\left(\gamma_{\xi^{\prime}}^{0}\right)} \exp \left(\frac{i}{2} \operatorname{rot}\left(\gamma_{\xi^{\prime}}\right)\right) x\left(\xi^{\prime}\right)\left(1-\sum_{\substack{e \in \xi^{\prime} \\ t\left(e_{1}\right) \in e \neq e_{1}}}(-1)^{n_{\xi^{\prime}}\left(e_{1}, e\right)}\right),
$$

which vanishes by Lemma 4.6 ,

Let us finally assume that $e_{1} \neq e_{2}$ and $t\left(e_{1}\right)=o\left(e_{2}\right)=v$. This time, we have

$$
\sum_{e \in \mathbb{E}} K W\left(e_{1}, e\right) F\left(e, e_{2}\right)=\exp \left(\frac{i}{2} \alpha\left(e_{1}, e_{2}\right)\right) x_{e_{1}}\left(X_{1}+X_{2}-\sum_{\substack{\xi^{\prime} \in \mathscr{\xi} \\ e_{2} \notin \xi^{\prime}}}(-1)^{q\left(\xi^{\prime}\right)} x\left(\xi^{\prime}\right)\right),
$$

where

$$
X_{1}=\sum_{\xi \in \mathscr{E}\left(e_{1}, e_{2}\right)}(-1)^{q\left(\gamma_{\xi}^{0}\right)} \exp \left(\frac{i}{2}\left(-\alpha\left(e_{1}, e_{2}\right)+\operatorname{rot}\left(\gamma_{\xi}\right)\right)\right) x(\xi)
$$

and

$$
X_{2}=-\sum_{\substack{o(e)=v \\ e \neq \bar{e}_{1}, e_{2}}} \sum_{\xi \in \mathscr{E}\left(e, e_{1}\right)}(-1)^{q\left(\gamma_{\xi}^{0}\right)} \exp \left(\frac{i}{2}\left(-\alpha\left(e_{1}, e_{2}\right)+\alpha\left(e_{1}, e\right)+\operatorname{rot}\left(\gamma_{\xi}\right)\right)\right) x_{e} x(\xi) .
$$

Let us deal with the first term. Given $\xi \in \mathscr{E}\left(e_{1}, e_{2}\right)$, let $\xi^{\prime} \in \mathscr{E}$ (resp. $\left.\gamma_{\xi}^{\prime}\right)$ denote the 1cycle obtained from $\xi$ (resp. $\left.\gamma_{\xi}\right)$ by removing the half-edges $\left(z_{e_{1}}, v\right)$ and $\left(v, z_{e_{2}}\right)$. We have $x(\xi)=x\left(\xi^{\prime}\right)$, while

$$
\exp \left(\frac{i}{2}\left(-\alpha\left(e_{1}, e_{2}\right)+\operatorname{rot}\left(\gamma_{\xi}\right)\right)\right)=-\exp \left(\frac{i}{2} \operatorname{rot}\left(\gamma_{\xi}^{\prime}\right)\right)=(-1)^{q\left(\gamma_{\xi}^{\prime}\right)+t\left(\gamma_{\xi}^{\prime}\right)}=(-1)^{q\left(\xi^{\prime}\right)+n_{\xi^{\prime}}\left(e_{1}, e_{2}\right)}
$$

as in the first step of the proof. Therefore,

$$
X_{1}=\sum_{\substack{\xi^{\prime} \in \mathscr{E} \\ e_{1}, e_{2} \notin \xi^{\prime}}}(-1)^{q\left(\xi^{\prime}\right)+n_{\xi^{\prime}}\left(e_{1}, e_{2}\right)} x\left(\xi^{\prime}\right)
$$

Let us now deal with the second term. This time, let us assign to $\xi \in \mathscr{E}\left(e, e_{2}\right)$ the 1 -cycle $\xi^{\prime} \in \mathscr{E}$ obtained from $\xi$ by adding the half-edge $\left(v, z_{e}\right)$ and removing $\left(v, z_{e_{2}}\right)$. Clearly, $x_{e} x(\xi)=x\left(\xi^{\prime}\right)$. As before, we obtain

$$
-(-1)^{q\left(\gamma_{\xi}^{0}\right)} \exp \left(\frac{i}{2}\left(-\alpha\left(e_{1}, e_{2}\right)+\alpha\left(e_{1}, e\right)+\operatorname{rot}\left(\gamma_{\xi}\right)\right)\right)=(-1)^{q\left(\xi^{\prime}\right)+n_{\xi^{\prime}}\left(e_{1}, e\right)},
$$

so that

$$
X_{2}=\sum_{\substack{\xi^{\prime} \in \mathscr{G} \\ e_{2} \notin \xi^{\prime}}}(-1)^{q\left(\xi^{\prime}\right)} x\left(\xi^{\prime}\right)\left(\sum_{\substack{v \in e \in \xi^{\prime} \\ e \neq \bar{e}_{1}, e_{2}}}(-1)^{n_{\xi^{\prime}}\left(e_{1}, e\right)}\right)
$$


Using Lemma 4.6 one last time, we get

$$
X_{1}+X_{2}=\sum_{\substack{\xi^{\prime} \in \mathscr{E} \\ e_{2} \notin \xi^{\prime}}}(-1)^{q\left(\xi^{\prime}\right)} x\left(\xi^{\prime}\right)\left(\sum_{\substack{v \in e \in \xi^{\prime} \\ e \neq \bar{e}_{1}}}(-1)^{n_{\xi^{\prime}}\left(e_{1}, e\right)}\right)=\sum_{\substack{\xi^{\prime} \in \mathscr{E} \\ e_{2} \notin \xi^{\prime}}}(-1)^{q\left(\xi^{\prime}\right)} x\left(\xi^{\prime}\right) .
$$

Therefore, this coefficient vanishes as well, and the proof is completed.

This theorem immediately provides us with a wealth of s-holomorphic functions. These generalize the spin-Ising fermionic observables of [6] and of [4, which correspond to the case of critical isoradial graphs embedded in simply-connected planar domains, and critical square lattices embedded in multiply-connected planar domains, respectively.

Corollary 4.7. Given any fixed oriented edge $e_{0} \in \mathbb{E}$, the function $F_{e_{0}} \in \mathbb{C}^{\diamond}$ defined by

$$
F_{e_{0}}(z)=\frac{\exp \left(i \frac{\pi}{4}\right)}{\cos \left(\theta_{e} / 2\right)} \sum_{\xi \in \mathscr{E}\left(e_{0}, z\right)}(-1)^{q\left(\gamma_{\xi}^{0}\right)} \exp \left(-\frac{i}{2} \operatorname{rot}\left(\gamma_{\xi}\right)\right) x(\xi),
$$

where $z=z_{e} \neq z_{e_{0}}$ and $\mathscr{E}\left(e_{0}, z\right)=\mathscr{E}\left(e_{0}, e\right) \cup \mathscr{E}\left(e_{0}, \bar{e}\right)$, is s-holomorphic around every vertex of $\Gamma$ not adjacent to $e_{0}$.

Proof. First note that there is a bijection between $\mathscr{E}\left(e_{0}, z\right)$ and $\mathscr{E}\left(z, \bar{e}_{0}\right)=\mathscr{E}\left(e, \bar{e}_{0}\right) \cup \mathscr{E}\left(\bar{e}, \bar{e}_{0}\right)$ reversing the orientation of $\gamma_{\xi}$. Therefore, for $z=z_{e} \neq z_{e_{0}}$,

$$
F_{e_{0}}(z)=\frac{\exp \left(i \frac{\pi}{4}\right)}{\sin \left(\theta_{e} / 2\right)} \sum_{\xi \in \mathscr{E}\left(z, \bar{e}_{0}\right)}(-1)^{q\left(\gamma_{\xi}^{0}\right)} \exp \left(\frac{i}{2} \operatorname{rot}\left(\gamma_{\xi}\right)\right) x_{e} x(\xi)=\exp \left(i \frac{\pi}{4}\right)\left(S^{-1} f\right)(z),
$$

with $f \in \mathbb{C}^{\mathbb{E}}$ defined by $f(e)=F\left(e, \bar{e}_{0}\right)$. By Proposition 4.1, $F_{e_{0}}$ is s-holomorphic around a vertex $v$ if and only if $(K W f)(\bar{e})$ vanishes for all $\bar{e} \in \mathbb{E}_{v}$. For $v$ not adjacent to $e_{0}$, we have $(K W f)(\bar{e})=(K W \circ F)\left(\bar{e}, \bar{e}_{0}\right)=0$ by Theorem 4.5.

This theorem also has a direct link to the Ising model. Indeed, consider a locally finite planar graph $\mathscr{G}$ with edge weights $J=\left(J_{e}\right)_{e} \in(0, \infty)^{E(\mathscr{G})}$ and faces that are bounded topological discs. Let us assume that this weighted graph is biperiodic, i.e. invariant under the action of a lattice $L \simeq \mathbb{Z}^{2}$.

Corollary 4.8. There exists a non-trivial biperiodic s-holomorphic function on the weighted graph $(\mathscr{G}, x)$, where $x_{e}=\tanh \left(\beta J_{e}\right)$, if and only if $\beta$ is the critical inverse temperature for the Ising model on $(\mathscr{G}, J)$.

Proof. A biperiodic weighted graph $(\mathscr{G}, x)$ as above is equivalent to a finite weighted surface graph $(\Gamma, x)=(\mathscr{G} / L, x)$ embedded in the torus $\mathbb{C} / L$. In the recent paper [10], Duminil-Copin and the author identified the critical inverse temperature $\beta_{c}$ for the Ising model on $(\mathscr{G}, J)$ as the only value of $\beta$ such that $\operatorname{det}\left(K W^{\lambda}(\Gamma, x)\right)$ vanishes. The result therefore follows from Theorems 4.2 and 4.5 .

4.4. The integral of $F^{2}$. The aim of this last paragraph is to show that one of the most remarkable facts discovered in [6] extends to our setting: the existence of a discrete version of $\operatorname{Im} \int f(z)^{2} d z$ for s-holomorphic functions. 
Let us fix a weighted surface graph $(\Gamma, x) \subset \Sigma$ with weights parametrized by $x_{e}=\tan \left(\theta_{e} / 2\right)$, and let us assume that dual edges $e$ and $e^{*}$ meet orthogonally, so that $D_{e^{*}}=i D_{e}$. Given an s-holomorphic function $F \in \mathbb{C}^{\diamond}$, define $H \in \mathbb{C}^{\Lambda}=\mathbb{C}^{V(\Gamma)} \oplus \mathbb{C}^{V\left(\Gamma^{*}\right)}$ by

$H(v)-H(w):=2\left|\operatorname{Pr}\left(F(z) ;\left[i \exp \left(i\left(a_{e}+\theta_{e}\right)\right)\right]^{-\frac{1}{2}}\right)\right|^{2}=2\left|\operatorname{Pr}\left(F\left(z^{\prime}\right) ;\left[i \exp \left(i\left(a_{e^{\prime}}-\theta_{e^{\prime}}\right)\right)\right]^{-\frac{1}{2}}\right)\right|^{2}$ for $v \in V(\Gamma)$ and $w \in V\left(\Gamma^{*}\right)$, with the notations illustrated below. Note that the equality above follows from the very definition of s-holomorphicity.

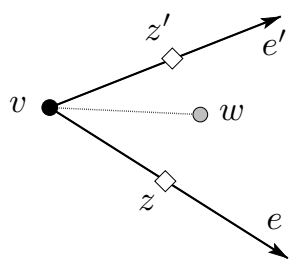

Proposition 4.9. The restriction of $H \in \mathbb{C}^{\Lambda}$ to any simply connected domain in $\Sigma$ is welldefined up to an additive constant. Moreover, it satisfies the equation

$$
H\left(v_{2}\right)-H\left(v_{1}\right)=\operatorname{Im}\left(2 \cos \left(\theta_{e}\right) D_{e} F\left(z_{e}\right)^{2}\right)
$$

for any $v_{1}, v_{2} \in V(\Gamma)$ (resp. $\left.V\left(\Gamma^{*}\right)\right)$ linked by $e=\left(v_{1}, v_{2}\right) \in \mathbb{E}(\Gamma)$ (resp. $\left.\mathbb{E}\left(\Gamma^{*}\right)\right)$.

Proof. Fix $z=z_{e} \in \diamond$, and let $\theta=\theta_{e}$ denote the corresponding angle. Also, let $\left(v_{1}, w_{1}, v_{2}, w_{2}\right)$ denote the four vertices in $\Lambda$ around $z$ in counterclockwise order with $e=\left(v_{1}, v_{2}\right) \in \mathbb{E}(\Gamma)$, so that $e^{*}=\left(w_{1}, w_{2}\right) \in \mathbb{E}\left(\Gamma^{*}\right)$. By definition of $H$ and of $D_{e}$, we have

$$
\begin{aligned}
H\left(v_{2}\right)- & H\left(v_{1}\right)=\left(H\left(v_{2}\right)-H\left(w_{2}\right)\right)-\left(H\left(v_{1}\right)-H\left(w_{2}\right)\right) \\
& =2\left|\operatorname{Pr}\left(F(z) ;\left[i \exp \left(i\left(a_{\bar{e}}-\theta\right)\right)\right]^{-1 / 2}\right)\right|^{2}-2\left|\operatorname{Pr}\left(F(z) ;\left[i \exp \left(i\left(a_{e}+\theta\right)\right)\right]^{-1 / 2}\right)\right|^{2} \\
& =2 \operatorname{Re}\left((i \exp (-i \theta))^{1 / 2} D_{\bar{e}}^{1 / 2} F(z)\right)^{2}-2 \operatorname{Re}\left((i \exp (i \theta))^{1 / 2} D_{e}^{1 / 2} F(z)\right)^{2} \\
& =\frac{F(z)^{2}}{2}\left(i \exp (-i \theta) D_{\bar{e}}-i \exp (i \theta) D_{e}\right)+\frac{\overline{F(z)}^{2}}{2}\left(-i \exp (i \theta) \overline{D_{\bar{e}}}+i \exp (-i \theta) \overline{D_{e}}\right) \\
& =\cos (\theta)\left(-i D_{e} F(z)^{2}+i{\overline{D_{e}}}_{\overline{F(z)}}^{2}\right) \\
& =\operatorname{Im}\left(2 \cos (\theta) D_{e} F(z)^{2}\right),
\end{aligned}
$$

using the fact that $D_{\bar{e}}=-D_{e}$. A similar computation leads to

$$
\left(H\left(v_{2}\right)-H\left(w_{1}\right)\right)-\left(H\left(v_{1}\right)-H\left(w_{1}\right)\right)=\operatorname{Im}\left(2 \cos (\theta) D_{e} F(z)^{2}\right) .
$$

By simple connectivity, this shows that $H$ is well-defined on $V(\Gamma)$ up to an additive constant. Similarly, one computes

$$
\left(H\left(v_{1}\right)-H\left(w_{1}\right)\right)-\left(H\left(v_{1}\right)-H\left(w_{2}\right)\right)=\operatorname{Re}\left(2 \sin (\theta) D_{e} F(z)^{2}\right)
$$

and

$$
\left(H\left(v_{2}\right)-H\left(w_{1}\right)\right)-\left(H\left(v_{2}\right)-H\left(w_{2}\right)\right)=\operatorname{Re}\left(2 \sin (\theta) D_{e} F(z)^{2}\right) .
$$

Since $e^{*}=\left(w_{1}, w_{2}\right), \theta_{e^{*}}=\frac{\pi}{2}-\theta$ and $D_{e^{*}}=i D_{e}$, we have

$$
H\left(w_{2}\right)-H\left(w_{1}\right)=\operatorname{Im}\left(2 \cos \left(\theta_{e^{*}}\right) D_{e^{*}} F(z)^{2}\right) .
$$

This completes the proof. 
Let us conclude this section, and this paper, with one last remark and some comments about possible future work. In the isoradial case, it is showed in [6] that $H$ is discrete superharmonic on $\Gamma$ and subharmonic on $\Gamma^{*}$. More precisely, given any vertex $v \in V(\Gamma)$, let us denote by $\theta_{1}, \ldots, \theta_{n}$ the adjacent half-rhombus angles. Then,

$$
\left(\Delta_{\Gamma} H\right)(v)=-\frac{1}{\mu_{v}} Q_{\theta_{1} ; \ldots ; \theta_{n}}^{(n)}\left(t_{1}, \ldots, t_{n}\right),
$$

where $Q_{\theta_{1} ; \ldots ; \theta_{n}}^{(n)}$ is some explicit quadratic form depending on the parameters $\theta_{1}, \ldots, \theta_{n}$, and $t_{1}, \ldots, t_{n}$ are real numbers. For $\theta_{1}, \ldots, \theta_{n}>0$ with $\theta_{1}+\cdots+\theta_{n}=\pi$, Chelkak-Smirnov prove that this quadratic form is non-negative, which implies the claim.

It turns out that even in the most general case of an arbitrary weighted surface graph, the equality displayed above still holds, where $\Delta_{\Gamma}$ is the associated discrete Laplacian (Definition 2). Moreover, $Q_{\theta_{1} ; \ldots ; \theta_{n}}^{(n)}$ is easily seen to remain non-negative when the $\theta_{j}$ 's add up to an odd multiple of $\pi$. Therefore, $H$ remains discrete superharmonic on $\Gamma$ and subharmonic on $\Gamma^{*}$ when the weighted graph $(\Gamma, x)$ can be isoradially embedded in a flat surface (recall Definition (1) with the given weights $x$ coinciding with the corresponding critical ones. This could possibly lead to some conformal invariance results in the critical isoradial case in non-trivial topology. However, the quadratic form $Q_{\theta_{1} ; \ldots ; \theta_{n}}^{(n)}$ is obviously not non-negative for arbitrary (positive) values of the parameters $\theta_{1}, \ldots, \theta_{n}$. Therefore, the discrete superharmonicity of $H$ does not extend in full generality. In particular, the extension of the results of ChelkakSmirnov to arbitrary biperiodic graphs at criticality will require the use of another (less naive) discretization of the Laplacian.

\section{REFERENCES}

[1] Michael F. Atiyah. Riemann surfaces and spin structures. Ann. Sci. École Norm. Sup. (4), 4:47-62, 1971.

[2] Cédric Boutillier and Béatrice de Tilière. The critical $Z$-invariant Ising model via dimers: the periodic case. Probab. Theory Related Fields, 147(3-4):379-413, 2010.

[3] Cédric Boutillier and Béatrice de Tilière. The critical $Z$-invariant Ising model via dimers: locality property. Comm. Math. Phys., 301(2):473-516, 2011.

[4] Dmitry Chelkak and Konstantin Izyurov. Holomorphic Spinor Observables in the Critical Ising Model. Comm. Math. Phys., 322(2):303-332, 2013.

[5] Dmitry Chelkak and Stanislav Smirnov. Discrete complex analysis on isoradial graphs. Adv. Math., 228(3):1590-1630, 2011.

[6] Dmitry Chelkak and Stanislav Smirnov. Universality in the 2D Ising model and conformal invariance of fermionic observables. Invent. Math., 189(3):515-580, 2012.

[7] D. Cimasoni. A generalized Kac-Ward formula. J. Stat. Mech., page P07023, 2010.

[8] David Cimasoni. The critical Ising model via Kac-Ward matrices. Comm. Math. Phys., 316(1):99-126, 2012.

[9] David Cimasoni. Discrete Dirac operators on Riemann surfaces and Kasteleyn matrices. J. Eur. Math. Soc. (JEMS), 14(4):1209-1244, 2012.

[10] David Cimasoni and Hugo Duminil-Copin. The critical temperature for the ising model on planar doubly periodic graphs. Electron. J. Probab., 18:no. 44, 1-18, 2013.

[11] David Cimasoni and Nicolai Reshetikhin. Dimers on surface graphs and spin structures. I. Comm. Math. Phys., 275(1):187-208, 2007.

[12] Ruben Costa-Santos and Barry M. McCoy. Finite size corrections for the Ising model on higher genus triangular lattices. J. Statist. Phys., 112(5-6):889-920, 2003.

[13] Béatrice de Tilière. Quadri-tilings of the plane. Probab. Theory Related Fields, 137(3-4):487-518, 2007. 
[14] N. P. Dolbilin, Yu. M. Zinov'ev, A. S. Mishchenko, M. A. Shtan'ko, and M. I. Shtogrin. The twodimensional Ising model and the Kac-Ward determinant. Izv. Ross. Akad. Nauk Ser. Mat., 63(4):79-100, 1999.

[15] Julien Dubédat. Exact bosonization of the Ising model. arXiv:1112.4399v1 [math.PR], December 2011.

[16] H. Duminil-Copin and S. Smirnov. Conformal invariance in lattice models. In D. Ellwood, C. Newman, V. Sidoravicius, and W. Werner, editors, Lecture notes, in Probability and Statistical Physics in Two and More Dimensions. CMI/AMS - Clay Mathematics Institute Proceedings, 2011.

[17] Beno Eckmann. Harmonische Funktionen und Randwertaufgaben in einem Komplex. Comment. Math. Helv., 17:240-255, 1945.

[18] Michael E. Fisher. On the dimer solution of planar Ising models. Journal of Mathematical Physics, $7(10): 1776-1781,1966$.

[19] Robin Forman. Determinants of Laplacians on graphs. Topology, 32(1):35-46, 1993.

[20] Anna Galluccio and Martin Loebl. On the theory of Pfaffian orientations. I. Perfect matchings and permanents. Electron. J. Combin., 6:Research Paper 6, 18 pp. (electronic), 1999.

[21] C. A. Hurst and H. S. Green. New solution of the Ising problem for a rectangular lattice. The Journal of Chemical Physics, 33(4):1059-1062, 1960.

[22] Dennis Johnson. Spin structures and quadratic forms on surfaces. J. London Math. Soc. (2), 22(2):365373,1980

[23] M. Kac and J. C. Ward. A combinatorial solution of the two-dimensional Ising model. Phys. Rev., 88:1332$1337,1952$.

[24] P. W. Kasteleyn. The statistics of dimers on a lattice. Physica, 27:1209-1225, 1961.

[25] P. W. Kasteleyn. Dimer statistics and phase transitions. J. Mathematical Phys., 4:287-293, 1963.

[26] P. W. Kasteleyn. Graph theory and crystal physics. In Graph Theory and Theoretical Physics, pages 43-110. Academic Press, London, 1967.

[27] Richard Kenyon. The Laplacian and Dirac operators on critical planar graphs. Invent. Math., 150(2):409439, 2002.

[28] Richard Kenyon. Spanning forests and the vector bundle Laplacian. Ann. Probab., 39(5):1983-2017, 2011.

[29] Richard Kenyon, Andrei Okounkov, and Scott Sheffield. Dimers and amoebae. Ann. of Math. (2), 163(3):1019-1056, 2006.

[30] Richard Kenyon, Nike Sun, and David Wilson. On the asymptotics of dimers on tori. arXiv:1310.2603 [math-ph], October 2013.

[31] H. A. Kramers and G. H. Wannier. Statistics of the two-dimensional ferromagnet. I. Phys. Rev. (2), 60:252-262, 1941

[32] W. Lenz. Beitrag zum verständnis der magnetischen eigenschaften in festen körpern. Phys. Zeitschr., 21:613-615, 1920.

[33] Zhongyang Li. Critical temperature of periodic Ising models. Comm. Math. Phys., 315(2):337-381, 2012.

[34] Marcin Lis. The fermionic observable in the Ising model and the inverse Kac-Ward operator. arXiv:1303.3017 [math-ph], March 2013.

[35] Marcin Lis. Phase transition free regions in the Ising model via the Kac-Ward operator. arXiv: 1306.2253 [math-ph], June 2013.

[36] Christian Mercat. Discrete Riemann surfaces and the Ising model. Comm. Math. Phys., 218(1):177-216, 2001.

[37] Lars Onsager. Crystal statistics. I. A two-dimensional model with an order-disorder transition. Phys. Rev. (2), 65:117-149, 1944.

[38] R. Peierls. On ising's model of ferromagnetism. Math. Proc. Camb. Phil. Soc., 32:477-481, 1936.

[39] Jean-Pierre Serre. Arbres, amalgames, SL2. Société Mathématique de France, Paris, 1977. Avec un sommaire anglais, Rédigé avec la collaboration de Hyman Bass, Astérisque, No. 46.

[40] S. Sherman. Combinatorial aspects of the Ising model for ferromagnetism. I. A conjecture of Feynman on paths and graphs. J. Mathematical Phys., 1:202-217, 1960.

[41] Glenn Tesler. Matchings in graphs on non-orientable surfaces. J. Combin. Theory Ser. B, 78(2):198-231, 2000 .

[42] Marc Troyanov. Les surfaces euclidiennes à singularités coniques. Enseign. Math. (2), 32(1-2):79-94, 1986.

[43] B. L. van der Waerden. Die lange Reichweite der regelmassigen Atomanordnung in Mischkristallen. $Z$. Physik, 118:473-488, 1941. 
[44] F. Y. Wu and K. Y. Lin. Staggered ice-rule vertex model ${ }^{-}$the pfaffian solution. Phys. Rev. B, 12:419-428, Jul 1975.

Section de mathématiques, 2-4 Rue du Lièvre, 1211 Genève 4, Switzerland

E-mail address: David.Cimasoni@unige.ch 